University of Puget Sound

Sound Ideas

All Faculty Scholarship

Faculty Scholarship

$11-1-1988$

\title{
The division of the Martian eccentricity from Hipparchos to Kepler: A history of the approximations to Kepler motion
}

James C. Evans

University of Puget Sound, jcevans@pugetsound.edu

Follow this and additional works at: http://soundideas.pugetsound.edu/faculty_pubs

\section{Citation}

Evans, James C. 1988. "The Division of the Martian Eccentricity from Hipparchus to Kepler - a History of the Approximations to Kepler Motion." American Journal Of Physics 56(11): 1009-1024.

This Article is brought to you for free and open access by the Faculty Scholarship at Sound Ideas. It has been accepted for inclusion in All Faculty Scholarship by an authorized administrator of Sound Ideas. For more information, please contact soundideas@pugetsound.edu. 


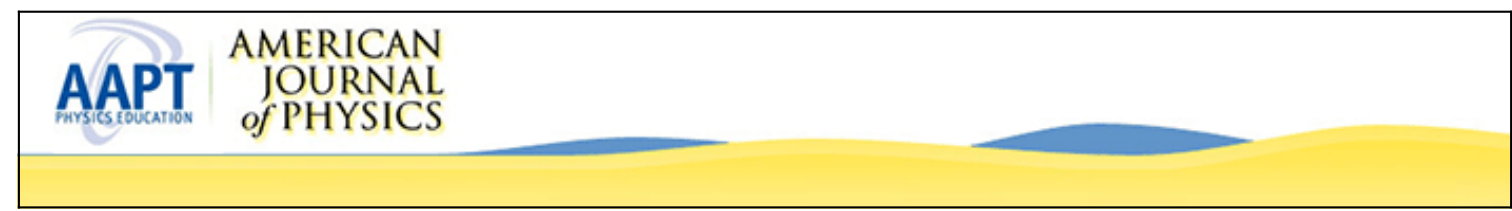

The division of the Martian eccentricity from Hipparchos to Kepler: A history of the approximations to Kepler motion

James Evans

Citation: American Journal of Physics 56, 1009 (1988); doi: 10.1119/1.15383

View online: http://dx.doi.org/10.1119/1.15383

View Table of Contents: http://scitation.aip.org/content/aapt/journal/ajp/56/11 ?ver=pdfcov

Published by the American Association of Physics Teachers

\section{Articles you may be interested in}

The great Martian catastrophe and how Kepler fixed it

Phys. Today 64, 50 (2011); 10.1063/PT.3.1259

Kepler's laws and Earth's eccentricity

Am. J. Phys. 57, 663 (1989); 10.1119/1.15934

History division is in the making

Phys. Today 34, 87 (1981); 10.1063/1.2914453

An Experiment for Studying Kepler's Laws and Planetary Masses from Satellite Motions

Am. J. Phys. 39, 1404 (1971); 10.1119/1.1976675

Simplified Treatment of Kepler Motion

Am. J. Phys. 38, 659 (1970); 10.1119/1.1976424

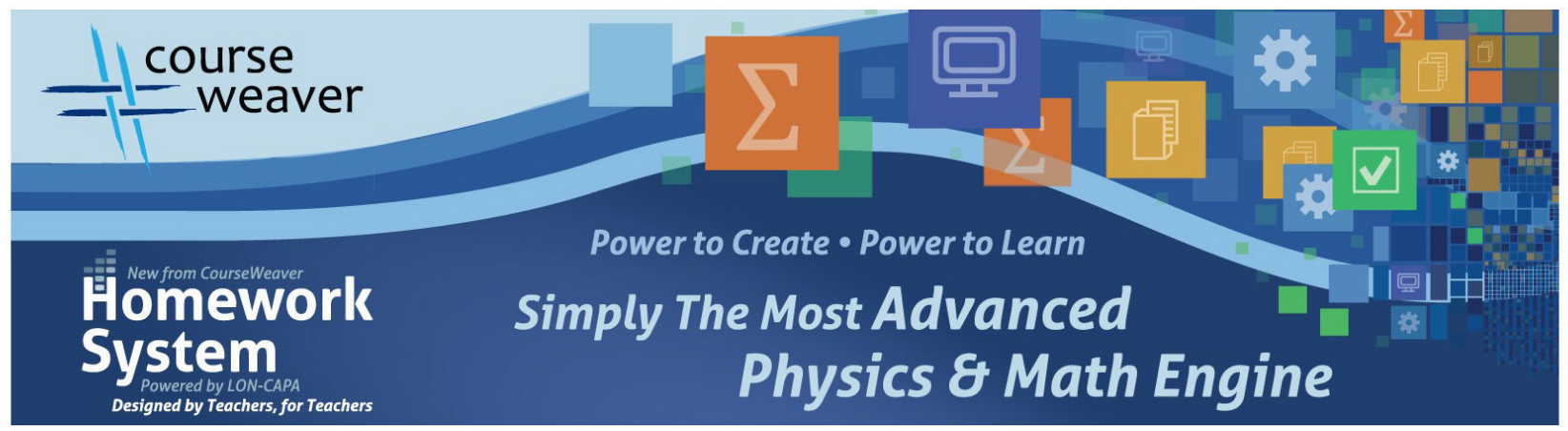


For the numbers chosen, $h=16.5, H=80$, and $2 D=6.4 \mathrm{~cm}$, the bow looks, experimentally, like a circle. But all that we actually calculated above was the intersection $x_{\mathrm{B}}$ of the bow with the $x$ axis, which we then called the radius. If we direct our eyes along the $y$ axis, instead of along the $x$ axis, and calculate the intersection of the perceived bow with the $y$ axis at $y=y_{\mathrm{B}}$ for the same height $z_{\mathrm{B}}=20.77$ as found above, then I calculate $y_{\mathrm{B}}=6.566 \mathrm{~cm}$, which is essentially the same as the result $x_{B}=6.567 \mathrm{~cm}$ found above. (I leave the formulas to the reader.) So the bow is indeed very close to being a circle. However, that is partly an accident of my eye separation. If I take the "wide eyed" $2 D=12.8 \mathrm{~cm}$, then, for $h=16.5$ and $H=80$, I find $x_{\mathrm{B}}=6.58 \mathrm{~cm}$ but $y_{\mathrm{B}}=5.07 \mathrm{~cm}$. Thus the perceived bow is ellipselike, wiith major axis along the line connecting the two eyes.

If your eyes are not directly above the light source, the bow is strongly ellipselike rather than circlelike, and is "tilted" relative to the $x-y$ plane. It is very pretty. But I have not tried to find the equations.

\section{ACKNOWLEDGMENTS}

I thank Charles Wohl and Richard Muller for helpful comments and suggestions, Cynthia Broderson and Larry
Webster for providing me with my first sample of Rainbow Dust, and Jack Navone, of Potters Industries, Inc., for a sample of Glory Dust.

'Highway Safety Spheres, Type 11 M.P. 0587 VG RG, index $n=1.51$, manufactured by Potters Industries Inc, Anaheim, CA. Sold retail at about $\$ 25$ per $50 \mathrm{lbs}$ at traffic and roadway suppliers all over the country, for example, at Hawkins Safety Products, 1255 E. Bayshore, Berkeley, CA. Potters also makes airport runway safety spheres with index $n=1.9$.

${ }^{2}$ C. B. Boyer, The Rainbow (Princeton, U.P., Princeton, NJ, 1987), a magnificent historical and theoretical study relating the early results of Descartes, and then of Isaac Newton, Jean Bernoulli, Edmund Halley, Jacob Hermann, and others.

${ }^{3}$ H. M. Nussenzveig, Sci. Am. 236(4), 116 (1977).

${ }^{4} \mathrm{M}$. Minnaert, The Nature of Light and Colour in the Open Air (Dover, New York, 1954), Chap. X.

5J. D. Walker, Am. J. Phys. 44, 421 (1976).

${ }^{6}$ Manufactured by MAG INSTRUMENT, Ontario, California.

${ }^{7}$ H. C. Bryant and N. Jarmie, Sci. Am. 231(7), 60 (1974).

${ }^{8}$ J. Walker, The Flying Circus of Physics (Wiley, New York, 1975).

${ }^{9}$ R. G. Greenler, Rainbows, Halos, and Glories (Cambridge U.P., Cambridge, 1980).

\title{
The division of the Martian eccentricity from Hipparchos to Kepler: A history of the approximations to Kepler motion
}

\author{
James Evans \\ Department of Physics, University of Puget Sound, Tacoma, Washington 98416
}

(Received 13 July 1987; accepted for publication 17 January 1988)

\begin{abstract}
Any planetary theory with pretensions of quantitative validity must grapple with the inequality of movement due to Kepler motion. This was so in antiquity no less than today. The technical details of six historically important planetary models are discussed. The relationships of the models to one another and to the real motions of the planets are examined with simple geometrical techniques. The discussion centers on the history of the attempts to provide a model for the motion of Mars.
\end{abstract}

\section{INTRODUCTION}

In roughest approximation, the planets may be considered to travel at constant speed upon circular orbits centered on the Sun. This model of planetary motion may be designated the "zero-eccentricity model," as it corresponds to Kepler motion in the limit of an ellipse of zero eccentricity. The uniform circular motion of the zero-eccentricity model lends itself to elementary instruction in physics and astronomy; it serves well for many order-ofmagnitude calculations. But it does not agree with the motions of the planets. Already in the fifth century B.C., the departure of the Sun from the zero-eccentricity model became apparent in the unequal lengths of the four seasons.
In the case of Mars, Kepler motion produces a striking inequality in the widths and spacings of the planet's retrograde arcs. This inequality was not only known in antiquity, but appears to have played an important part in Ptolemy's discovery of the equant. No planetary theory with pretensions of quantitative validity can fail to deal with the inequalities due to Kepler motion. Hipparchos' eccentric circle model for the Sun, Ptolemy's equant point, Copernicus' minor epicycle, and the "vicarious hypothesis" that Kepler employed before his discovery of the law of areas and the ellipticity of the orbits-all these represent historically important efforts to grapple with the departures of the planets from the simple motions of the zero-eccentricity model. 

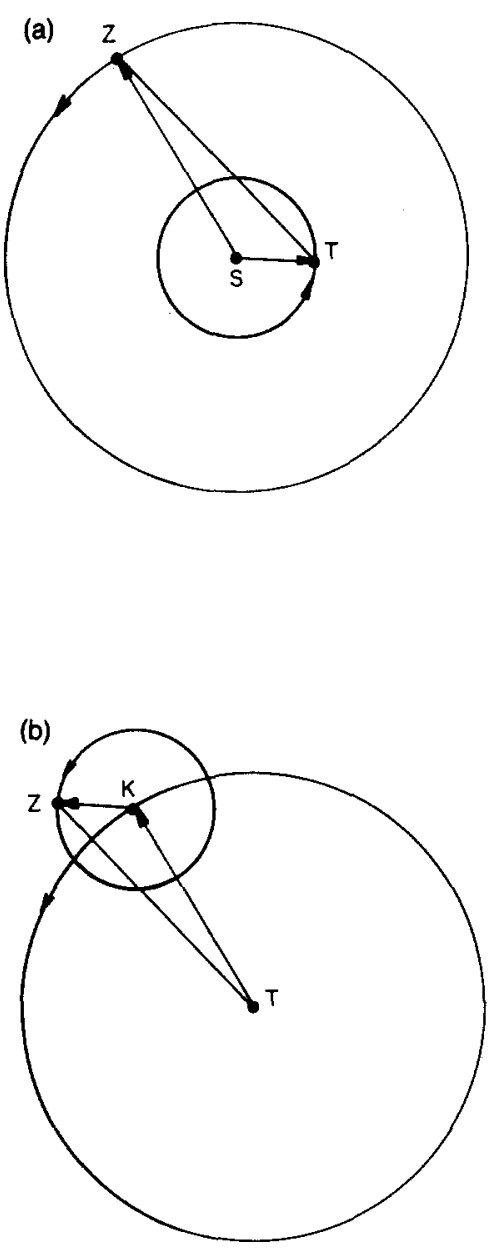

Fig. 1. Transformation from a heliocentric (a) to a geocentric (b) model. In this and the following figures, the plane of the ecliptic is viewed from above its north pole.

\section{A. Point of view}

Some of these attempts were couched in terms of geocentric models, others in terms of heliocentric models. However, the choice of Earth or Sun as center is of no consequence for our purposes. This may be demonstrated very simply. Figure 1 (a) represents the heliocentric theory of a superior planet. The Earth $T$ executes an orbit, not necessarily circular, about the stationary Sun $S$. In the course of a year, the position vector ST rotates counterclockwise about $S$. The angular speed of ST varies slightly in the course of the year, as does the length of the vector. Similar1 y, a superior planet $Z$ (Mars, perhaps) orbits the Sun in a larger orbit. Vector SZ turns at its own slightly varying rate. At any instant, the line of sight from the Earth to planet $Z$ coincides with vector $\mathbf{T Z}$, which is equal to $-\mathbf{S T}+\mathbf{S Z}$. Now, these vectors may be added in either order. Thus $\mathbf{T Z}$ is also equal to $\mathbf{S Z}+(-\mathbf{S T})$. The new form of the addition is shown in Fig. 1 (b). Here we begin at the Earth $T$. A vector equal to $\mathbf{S Z}$ is drawn with its tail at T; let the head of this vector be called $K$. Then TK rotates in Fig. 1(b) at the same rate as SZ rotates in Fig. 1(a). At $K$, place the tail of a second vector, equal to - ST. At the head of this vector is the planet $Z$. The result of this transformation is clear: If Fig. 1(a) represents the heliocentric theory of a superior planet, Fig. 1 (b) is the geocentric theory of the same planet. Point $K$ serves as the center of an epicycle upon which $Z$ revolves while $K$ itself moves on a deferent about the Earth $T$. The equivalence of Figs. 1(a) and $1(b)$ is a consequence of the commutative property of vector addition. Thus, in the case of a superior planet, the epicycle corresponds to the orbit of the Earth about the Sun; and the deferent, to the heliocentric orbit of the planet itself. (For inferior planets, the correspondences are reversed.)

The quality of a planetary model, then, does not depend on whether the Sun or the Earth is supposed to be stationary, which is merely a question of point of view. Rather, the issue is how well the hypotheses of the model - the shapes and positions of the orbits, and the rule governing the variation in speed-approximate the facts of Kepler motion.

\section{B. Outline of the article}

In Sec. II, six planetary models of historical significance are introduced. The goal of this article is to demonstrate, by geometrical arguments much simpler than those in common use, the relation of the older planetary models to Kepler motion. In Secs. III and IV we take up the question of how the most critical numerical parameter-the eccentricity-must be chosen to obtain the best agreement between the models and the real motion of the planets. A technical Appendix discusses the six models in terms of expansions in powers of th eccentricity. Throughout, the discussion centers on the history of the attempts to provide a model for the motion of Mars.

\section{THE MODELS}

\section{A. Kepler motion}

The planets move on ellipses (Fig. 2). At one focus is the Sun $O$. $C$ marks the center of the ellipse, and $F$ marks the empty focus. The line of apsides $O C F$ cuts the orbit at perihelion $P$ and aphelion $A$. The semimajor axis $C A$ or $C P$ is denoted $a$, and the semiminor axis $b$. If the eccentricity is denoted $e$, the distance of either focus from the center is $e a$. Finally, the planet's speed varies in such a way that the line from the Sun to the planet sweeps out equal areas in equal times.

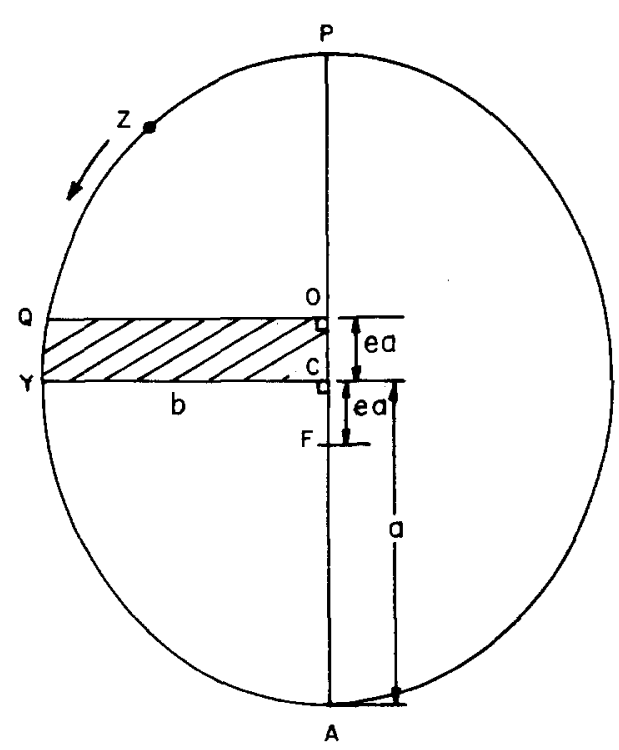

Fig. 2. Elliptical Kepler orbit. 
The eccentricity of Mars' elliptical orbit is roughly 0.09 . However, the eccentricity varies slightly with time because of perturbations of Mars' orbit by the other planets. More precise values for several epochs of interest are:

$\begin{array}{rrrr} & \text { Date } & e \text { (Mars) } \\ \text { AD } & 1900 & 0.0933 & \\ 1600 & 0.0930 & \begin{array}{c}\text { (appropriate for Copernicus, } \\ \text { Brahe, Kepler) }\end{array} \\ & 100 & 0.0916 & \begin{array}{c}\text { (appropriate for Ptolemy) } \\ \end{array}\end{array}$

\section{B. Eccentric circle}

In Fig. 3, a circular orbit $P Q A$ is described about center $C$. In the solar theory of Hipparchos, the Earth is stationary at $O$, while the Sun $Z$ moves at uniform speed around the circle. The Sun's circle is therefore said to be eccentric to the Earth. Let $r$ denote the radius of the circle. We define the Hipparchian eccentricity $e_{\mathbf{H}}$ as the distance between the Earth and the center of the circle, expressed in terms of the radius as unit. That is $e_{\mathrm{H}}=O C / r$. The line of apsides $O C$ cuts the circle at perigee $P$ and apogee $A$.

The eccentric circle was the standard theory of the Sun from the time of Hipparchos (second century B.C.) until 1609, when Kepler published the Astronomia nova. ${ }^{1} \mathrm{Co}-$ pernicus made the Earth travel uniformly on a circle eccentric to the Sun; but this shift in point of view had no bearing on the model's success as an approximation to Kepler motion.

The eccentric circle was also a standard tool of the planetary theory. Figure 4 represents the three stages of refinement through which the Greek deferent-and-epicycle theory passed. Figure 4(a) illustrates a zero eccentricity model. The planet $Z$ moves uniformly on a circular epicycle. The center $K$ of the epicycle moves uniformly around a deferent circle centered on the Earth $O$. The fixed reference line $O Y$ points to the vernal equinoctial point. Thus angles $\bar{\lambda}$ and $\bar{\mu}$ increase uniformly with time.

The zero-eccentricity model was discussed by Apollonios of Perge in the third century B.C. The model accounts in a qualitative way for the planets' retrogradations, and we

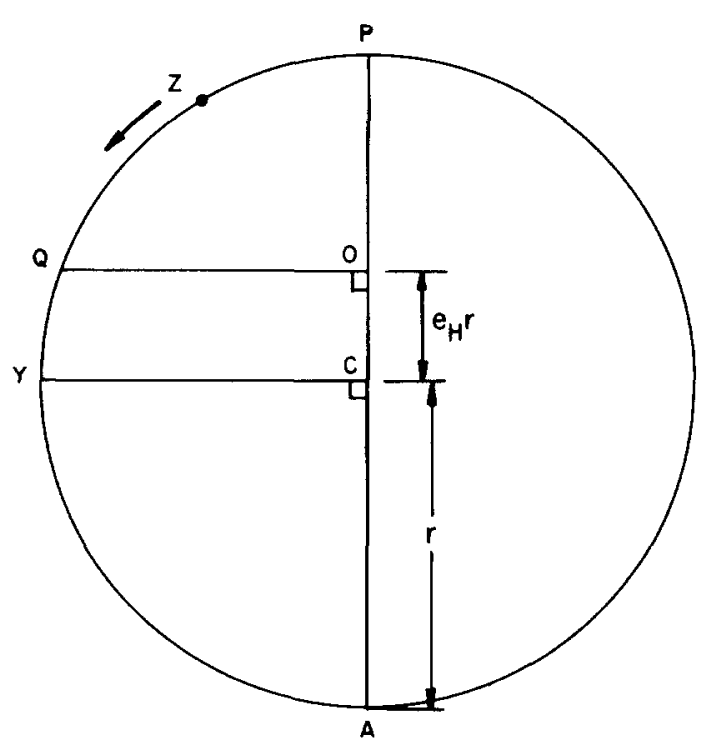

Fig. 3. Eccentric circle model. (a)

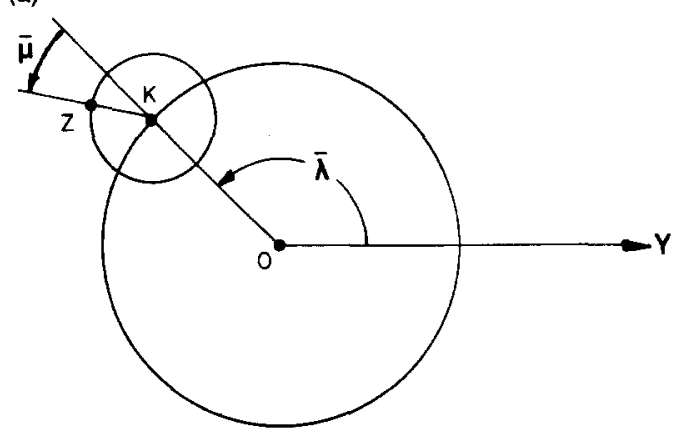

(b)

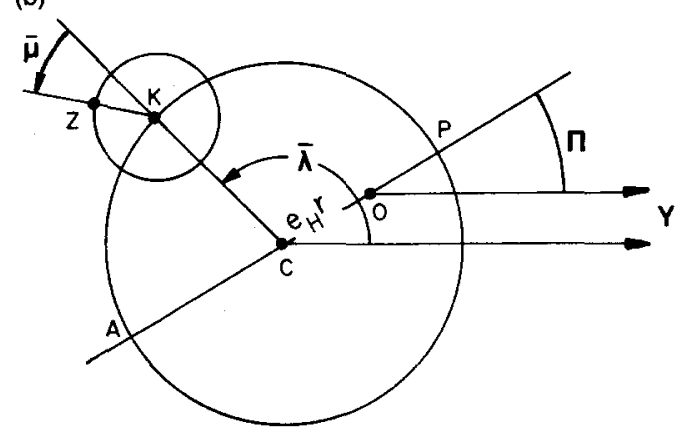

(c)

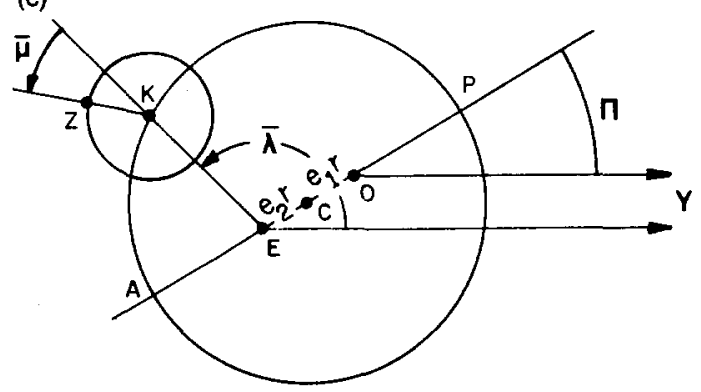

Fig. 4. The three stages of the Greek deferent and epicycle model: the zero-eccentricity model (a), the intermediate model (b), and the final Ptolemaic model (c).

know that Apollonios proved geometrical theorems involving retrograde motion in this model. Unfortunately, the zero-eccentricity model is quantitatively unworkable. It predicts, for example, that the retrograde arcs for a given planet will be equally spaced around the zodiac and all of the same length - which is very far from the truth. ${ }^{2}$ If $Z$ is a superior planet-say Mars--the epicycle corresponds to the orbit of the Earth about the Sun, and the deferent to Mars' heliocentric orbit. Now, the eccentricity of the Earth's orbit is small and the orbit is itself smaller than that of Mars; thus little harm results from the assumption of uniform circular motion for $Z$ on the epicycle. The serious defect is the zero-eccentricity motion of $K$ about $O$, which corresponds to a zero-eccentricity heliocentric motion for Mars.

Figure 4(b) represents an intermediate model, in which some account has been taken of the effects of Kepler motion in the deferent. The planet $Z$ moves uniformly on the epicycle. The center $K$ of the epicycle still moves around the deferent circle at uniform speed. But the center $C$ of the deferent is not located at the Earth $O$. If the eccentricity of 
the Earth's circular orbit can be neglected, the line of apsides $O C$ in the intermediate model should be parallel to the major axis of Mars' heliocentric ellipse. The purpose of the eccentricity of the deferent, then, is to account for the effects of Kepler motion in Mars' heliocentric orbit. The intermediate model introduces two new parameters whose numerical values must be determined for each planet. These are the eccentricity $e_{\mathbf{H}}$ of the deferent and the longitude II of the perigee. The intermediate model was in use by the time of Hipparchos ( second century B.C. ). Indeed, one of Hipparchos' contributions to astronomy was a demonstration that the intermediate model does not adequately represent the facts. ${ }^{3}$

\section{The Ptolemaic equant point}

Figure 4(c) represents the final stage of Greek planetary theory. The model shown was applied by Ptolemy to Venus, Mars, Jupiter, and Saturn. (Ptolemy's theory of Mercury has an extra complication.) The planet $Z$ (Mars, say) moves uniformly around an epicycle. The center $K$ of the epicycle moves upon a circular deferent whose center $C$ is eccentric to the Earth $O$. But now the motion of $K$ is assumed not to be uniform either with respect to the Earth or with respect to the center of the deferent. Rather, point $K$ moves in such a way that its angular speed appears constant if $K$ is observed from a third center $E$, the equant point. Thus the angles $\bar{\lambda}$ and $\bar{\mu}$ increase uniformly with time.

There are now two eccentricities to be defined. Let $r$ denote the radius of the deferent circle. The eccentricity of the Earth with respect to the center of the deferent is $e_{1}=O C /$ $r$. The eccentricity of the equant point is $e_{2}=E C / r$. The rule of equant motion-that $K$ move at constant angular speed as viewed from the equant - produces a physical variation in the speed of $K$. This variation in speed is determined by $e_{2}:$ As $e_{2}$ goes to zero, the motion of $K$ reduces to uniform circular motion. But even if $e_{2}$ were zero, the eccentricity $e_{1}$ of the Earth with respect to $C$ would cause the motion of $K$ to appear nonuniform from the Earth. This is, however, merely an optical effect, which depends on the position of the observer and does not involve a physical variation in speed. The sum $e_{1}+e_{2}$ is called the total eccentricity.

Ptolemy, in the theory of Venus and the three superior planets, always puts $e_{1}=e_{2}$. This situation is referred to as the bisection of the eccentricity. That is, half the total eccentricity is assigned to the Earth, and half to the equant. In further discussion of Ptolemy's model, we will refer simply to the Ptolemaic eccentricity $e_{\mathrm{P}}\left(=e_{1}=e_{2}\right)$. Ptolemy adopts the value $e_{\mathrm{p}}=0.1$ in the case of Mars. ${ }^{4}$ The total eccentricity $e_{1}+e_{2}$ is thus 0.2 .

Before leaving Ptolemy's model, we must indicate how the crucial numerical parameters-the eccentricity and the longitude of the perigee-may be determined from observations. Central to the procedure are the oppositions of the planet to the mean Sun. ${ }^{5}$ In Fig. 5(a), an observer on the Earth $T$ sees Mars $Z$ in opposition to the mean Sun $S$. Mars is in the middle of its apparent retrograde motion, since the Earth is passing by the planet. At this instant, an observer at $T$ sees $Z$ in the same direction as would an observer on the mean Sun $S$. Thus the use of oppositions allows the astronomer effectively to examine the motion of the planet from $S$.

Figure 5(b) illustrates the same situation in terms of a
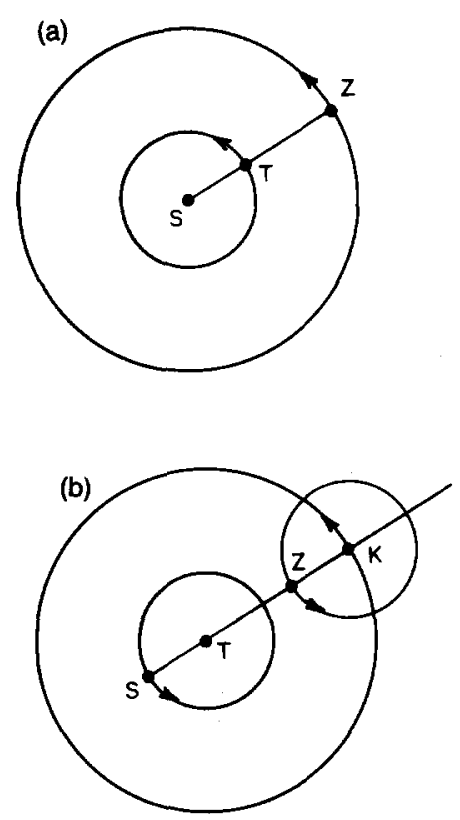

Fig. 5. Opposition of a superior planet $Z$ to the mean $S u n S$ in a heliocentric model (a) and a geocentric model (b).

geocentric model. The mean Sun $S$ travels uniformly on a circle centered on the Earth $T$. In the middle of retrograde motion, the planet $Z$ is at the perigee of its epicycle. Since $K Z$ is parallel to $T S$ (as indicated already in Fig. 1), it follows that, at opposition, $K, Z, T$, and $S$ are all collinear. Thus, at opposition, the observer on $T$ sees the planet $Z$ in the same direction as the epicycle's center $K$. The use of oppositions allows the observer effectively to ignore the motion on the epicycle and to follow the motion of $K$ around the Earth.

From the observed longitudes of the planet at three mean oppositions, and the time intervals between them, one may determine the eccentricity $e_{\mathrm{P}}$ and the longitude $\Pi$ of the perigee [Fig. 4(c), with $e_{1}=e_{2}=e_{\mathrm{P}}$ ]. The geometrical calculation is in all essentials the same whether one adopts a heliocentric or a geocentric point of view. ${ }^{6}$

\section{Copernican planetary theory}

The heliocentric theory of the Earth and Mars illustrated in Fig. 1(a) is, of course, only schematic: The theory is not complete without an explanation of the devices employed in the two orbits to account for the effects of Kepler motion.

For the orbit of the Earth, Copernicus chose an eccentric circle. Thus, in Fig. 1(a), $T$ is to be regarded as executing uniform circular motion, not about the Sun, but about a point in the vicinity of the Sun. The model is in essence the same as the solar theory of Hipparchos illustrated in Fig. 3.

For the other planets, Copernicus adopted a modified form of the Ptolemaic equant. The equant itself Copernicus held to be a physical and philosophical abomination. ${ }^{7}$ The only motion natural to the celestial bodies is uniform circular motion: From the time of Aristotle, this had been accepted physical principle. Ptolemy's equant point had stretched the definition of uniform circular motion past the breaking point. What sort of uniformity was this, when the motion was not uniform with respect to the center of the circular path? Copernicus rejected the equant but had, of 


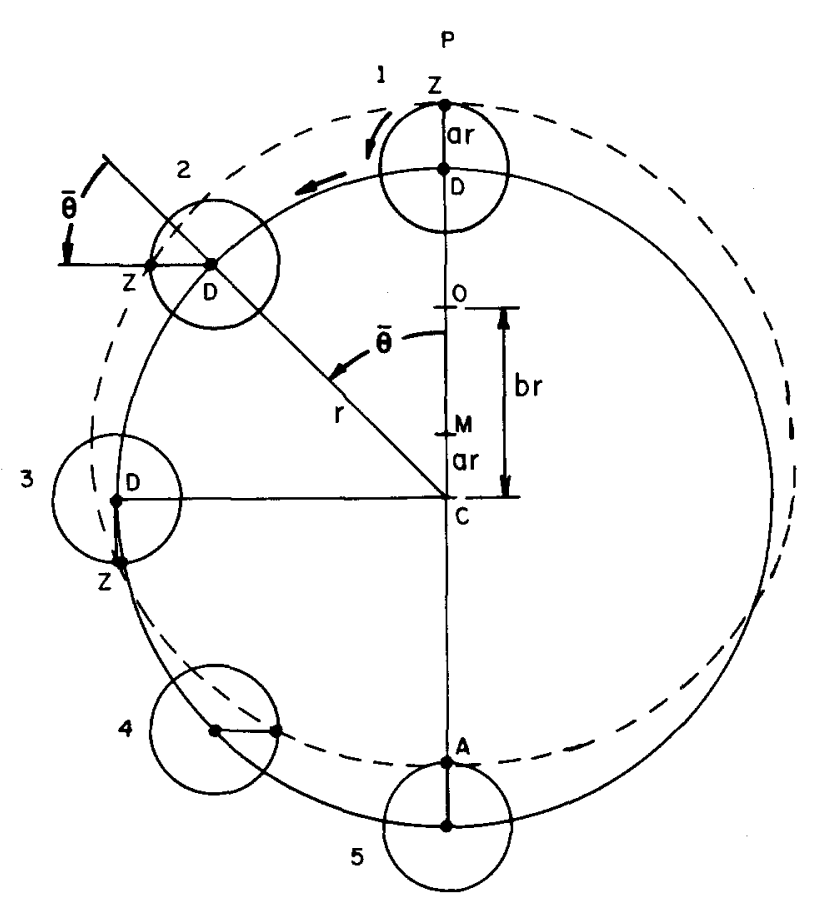

Fig. 6. Copernicus' minor epicycle, a replacement for Ptolemy's equant.

course, to replace it with something else. He found that a minor epicycle could perform very nearly the same function.

Refer to Fig. 6, which illustrates Copernicus' theory of Mars. The large, solid circle of radius $r$ represents the deferent of Mars, centered at $C$. The deferent circle is eccentric to $O$, the center of the Earth's orbit (also called the mean Sun ). The true Sun is in the vicinity of $O$ but plays no part in the theory. For this reason, Copernicus' theory has been aptly characterized as merely heliostatic, rather than truly heliocentric. The center of the Earth's orbit is the theoretical center of the whole system. The dimensionless eccentricity of the deferent circle is $b=\mathrm{CO} / r$.

The center $D$ of a small epicycle moves counterclockwise and uniformly around the deferent. The planet $Z$ moves counterclockwise and uniformly on the epicycle whose radius is $a r$. (Thus $a$ is a dimensionless number less than unity.) Further, the motion of $Z$ is such that the two angles marked $\bar{\theta}$ remain equal to one another while increasing uniformly with time. Consequently, while the epicycle's center moves through the $180 \mathrm{deg}$ from position 1 to position 5, the planet revolves through $360 \mathrm{deg}$ on the epicycle. ${ }^{8}$ It is important not to confuse Copernicus' minor epicycle with the large Ptolemaic epicycle of Fig. 4(c). Ptolemy's epicycle is responsible for retrograde motion. In the Copernican model, this function is assumed by the orbit of the Earth, not shown in Fig. 6. The minor epicycle of Fig. 6 replaces the Ptolemaic equant point.

The combination of two uniform circular motions for $Z$ in Fig. 6 results in a motion that is neither uniform nor circular. The actual path of the planet is indicated by the dashed line. The effective center of the orbit is not $C$ but $M$, located above $C$ by a distance $a r$ equal to one radius of the epicycle. As Copernicus himself explicitly states, the path is not circular but somewhat oblong-the long axis being perpendicular to the line of apsides $P O A .{ }^{9}$ That is, the long axis of the Copernican orbit coincides with the minor axis of the Keplerian ellipse. In this respect, Copernicus' model

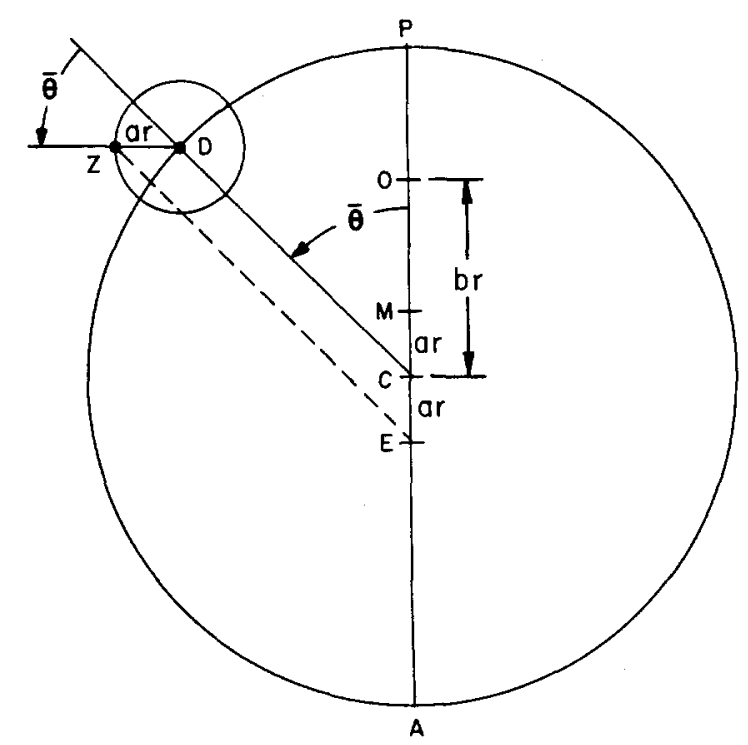

Fig. 7. Copernicus' hidden equant point.

represents a step backwards from Ptolemy's.

Nevertheless, Copernicus' speed rule is indistinguishable from Ptolemy's: The minor epicycle produces a motion that closely approximates equant motion. Refer to Fig. 7. The radius of the epicycle is $a r$. Let us identify point $E$ on the line of apsides at distance $a r$ below the center $C$ of the deferent. As already remarked, in Copernicus' model, the rotation of $D Z$ is such that the angle $C D Z$ is always equal to angle $A C D$ : Both are equal to $180^{\circ}-\bar{\theta}$. Since also $C E=D Z$, it follows that the quadrilateral $E C D Z$ is a trapezoid, with sides $E Z$ and $C D$ always parallel. Since line $C D$ turns uniformly, it follows that $E Z$ turns uniformly, too. In other words, $E$ is an effective equant point: The planet $Z$, observed from $E$, appears to move at absolutely uniform angular speed. ${ }^{10}$

Furthermore, Copernicus usually makes the radius of the minor epicycle exactly one-third the eccentricity of the deferent; that is, $b=3 a$. Now, from Fig. 7, $E M=2 a r$; and $M O=b r-a r$, so we get also $M O=2 a r$. Thus the center $M$ of the effective orbit is exactly midway between $O$ and the effective equant point $E$. Copernicus, like Ptolemy, bisects the total eccentricity: $E M=M O$ in Fig. 7, just as $E C=C O$ in Fig. 4(c). An almost perfect equivalence will be established between Ptolemy's eccentric circle with equant and Copernicus' eccentric circle with minor epicycle if we identify the radius of Copernicus' epicycle with half the Ptolemaic eccentricity; i.e., if $a=\frac{1}{2} e_{\mathrm{p}}$. And thus $b=\frac{3}{2} e_{\mathrm{P}}$.

If we wish to depart from Ptolemy and allow for the possibility that $e_{1}$ and $e_{2}$ are not the same, comparison of Fig. 4(c) and Fig. 7 gives the more general correspondences

$$
e_{1}=b-a, \quad e_{2}=2 a,
$$

or, equivalently,

$$
a=\frac{1}{2} e_{2}, \quad b=e_{1}+\frac{1}{2} e_{2} .
$$

The combined effect of Copernicus' oblong orbit and hidden equant is illustrated in Fig. 8. Here, $M$ is the center of the solid circle and $E$ represents a Ptolemaic equant point. Thus, if body $Z$ moves on the circle according to the law of the equant, $\bar{\theta}$ increases uniformly with time. The 


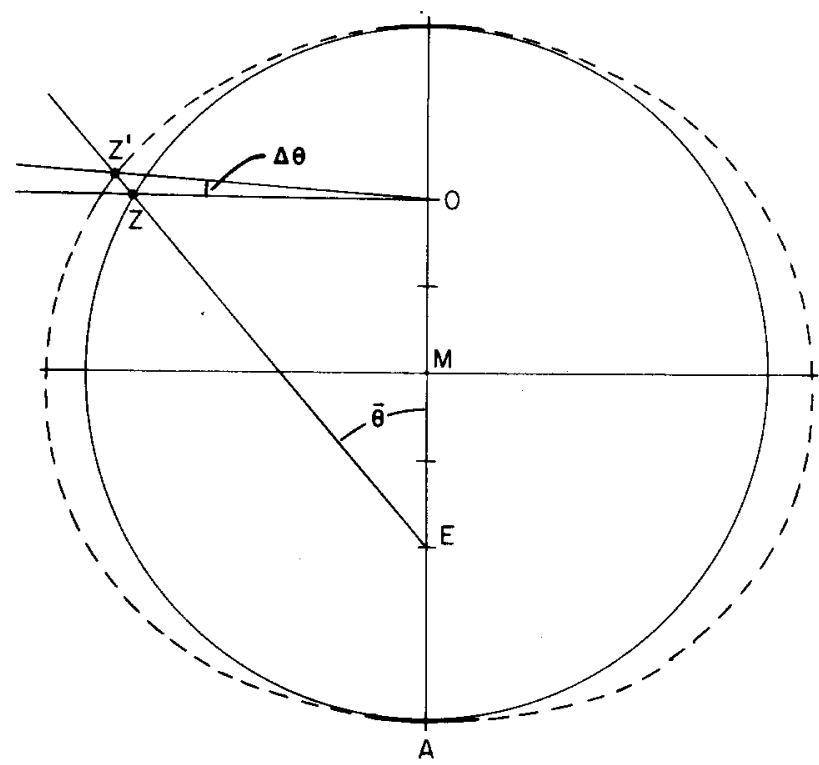

Fig. 8. Comparison of the Copernican model with a Ptolemaic eccentric with equant model.

dashed curve represents the effective, oblong Copernican orbit. $E$, then, is also the effective equant of the Copernican orbit. Thus, when the body is at $Z$ according to Ptolemaic hypotheses, it will be found at $Z^{\prime}$ according to Copernican principles: $Z^{\prime}$ must lie on line $E Z$. For an observer at the equant, $Z$ and $Z^{\prime}$ could not be distinguished. But because of the noncircularity of the Copernican orbit, an observer at $O$ (representing either the Earth or the mean Sun, as one wishes) will see $Z$ and $Z^{\prime}$ in directions that differ by a small angle $\Delta \theta$. The eccentricity has been greatly exaggerated in Fig. 8. It may be shown that the maximum size of $\Delta \theta$ is proportional to $e_{\mathrm{P}}^{3}$. Even in the case of Mars, for which $e_{\mathrm{p}}=0.1$, the maximum difference $\Delta \theta$ between the directions of $Z$ in the two models is only about $3^{\prime} .{ }^{11}$ Before the work of Brahe and Kepler, the observational consequences of Copernicus' modification of the Ptolemaic equant were nil. ${ }^{12}$

Moreover, Copernicus' values for the eccentricities of the superior planets were simply borrowed from Ptolemy, as may be seen in Table I, where $e_{\mathrm{P}}$ is Ptolemy's value for the eccentricity. As shown above, Copernicus' model differs insignificantly from Ptolemy's if $b=\frac{3}{2} e_{\mathrm{P}}$ and $a=\frac{1}{2}$ $e_{\mathrm{P}}$. The columns headed $\frac{3}{2} e_{\mathrm{P}}$ and $\frac{1}{2} e_{\mathrm{P}}$ give the appropriate fractions of Ptolemy's eccentricity. The columns headed $a$ and $b$ give the values of these parameters adopted by Copernicus in De revolutionibus. In the case of Jupiter and Saturn, the Copernican values are merely rounded versions of the Ptolemaic parameters. ${ }^{13}$ Only in the case of Mars did Copernicus make a slight change. Believing it necessary to reduce the total eccentricity of Mars slightly below its Ptolemaic value, Copernicus decided to leave the radius $a$ of the epicycle unchanged and to effect the reduction wholly in the eccentricity $b$ of the deferent. In the theory of Mars, then, Copernicus may be considered to have departed from a bisection of the total eccentricity: Equivalent eccentricities in an equant model for Mars would be $e_{1}=b-a=0.096$; and $e_{2}=2 a=0.100$. This minute departure from a bisection was without theoretical consequence and, indeed, was not required by the observational material at Copernicus' disposal. ${ }^{14}$
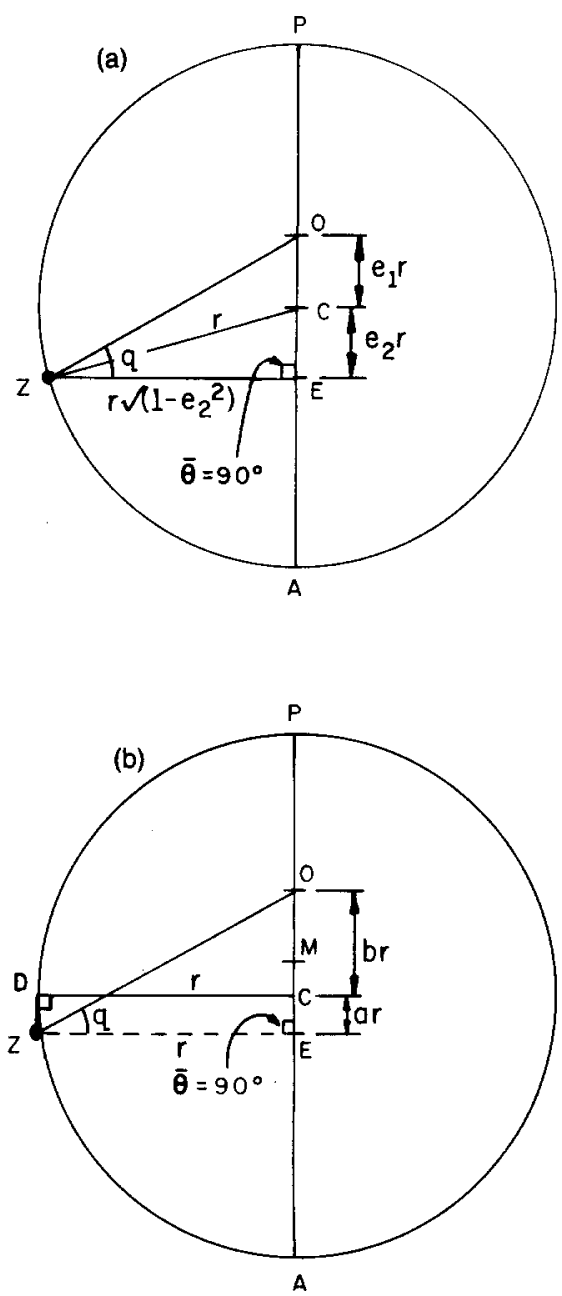

Fig. 9. The equation of center evaluated at $90^{\circ}$ of mean anomaly in the eccentric-with-equant model (a) and in the roughly equivalent Copernican model (b).

\section{E. Tycho Brahe's theory of Mars}

Tycho Brahe considered the Earth to be at rest. He admitted, however, the great explanatory power of a heliocentric theory. Accordingly, for Brahe, the five naked-eye planets all orbit the Sun, while the Sun revolves around the Earth. Geometrically, this is but a trivial transformation of Copernicus' system. In his choice of theoretical tools, Brahe was again a follower of Copernicus: To save the inequality in Mars' motion about the Sun, Brahe employed the minor epicycle of 'Copernicus rather than the equant point of Ptolemy.

When Kepler arrived in Prague as Brahe's guest early in the year 1600, he found Brahe's principal assistant, Longo-

Table I. Eccentricities of the superior planets according to Ptolemy and Copernicus.

\begin{tabular}{lccccc}
\hline \hline & $e_{\mathrm{P}}$ & $e_{\mathrm{P}} / 2$ & $\frac{3}{2} e_{\mathrm{P}}$ & $a$ & $b$ \\
\hline Mars & 0.10000 & 0.05000 & 0.15000 & 0.05000 & 0.14600 \\
Jupiter & 0.04583 & 0.02292 & 0.06875 & 0.02290 & 0.06870 \\
Saturn & 0.05694 & 0.02847 & 0.08541 & 0.02850 & 0.08540 \\
\hline \hline
\end{tabular}


montanus ${ }^{15}$ occupied with Mars. Brahe and Longomontanus had worked out a theory of Mars based on the Copernican model of Fig. 6. The total eccentricity and the direction of the line of apsides had been determined from three oppositions of Mars to the mean Sun. The model was checked against the observations of ten successive oppositions from 1580 to 1600 . Brahe and Longomontanus, however, had found it necessary to divide the eccentricity differently than had Copernicus. ${ }^{16}$

Indeed, Brahe and Longomontanus put

$$
a=0.0378, \quad b=0.1638 \text {, }
$$

so that $b / a=\frac{13}{3}$, rather than $\frac{9}{3}$ as in Copernicus. The total eccentricity $(a+b)$ came to 0.2016 , which differed but little from the values adopted by Ptolemy and Copernicus. The equivalent values of $e_{1}$ and $e_{2}$ in an equant model are

$$
\begin{aligned}
& e_{1}=b-a=0.1260, \\
& e_{2}=2 a=0.0756,
\end{aligned}
$$

so that $e_{1} / e_{2}=\frac{5}{3}$. To speak in Ptolemaic terms, while Ptolemy and Copernicus had assigned half of the total eccentricity to each $e_{1}$ and $e_{2}$, Brahe and Longomontanus gave $\frac{5}{8}$ of the total to $e_{1}$ and $\frac{3}{8}$ to $e_{2}$. Brahe and Longomontanus found that their theory agreed with the observed longitudes of the ten mean oppositions within 2 min of arc.

As we shall see below, a bisection of the eccentricity would have been incapable of such excellent agreement with the oppositions, a fact that Brahe and Longomontanus must certainly have discovered before their adoption of the unequal division. The 5:3 division on which they settled is, in fact, the best possible division for matching the kind of observation they were using. As their equivalent values for $e_{1}$ and $e_{2}$ stand exactly in the ratio $\frac{5}{3}$, it is clear that this division was the result of an a priori decision, but it probably was arrived at only after several trials.

Although the theory seemed to be in perfect accord with the observed longitudes of the ten mean oppositions, it failed completely to give the proper latitudes at opposition. Nor did it give satisfactory longitudes in situations away from the oppositions. And there Longomontanus was stuck.

\section{F. Kepler's vicarious hypothesis}

In his struggle with Mars, Kepler was guided constantly by physical principles, such as he perceived them. ${ }^{17}$ The book in which he recorded these struggles, and in which he announced the ellipticity of the orbit and the law of areas, was entitled, significantly, Astronomia nova AITIO-

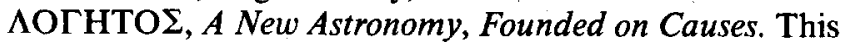
new insistence upon physical causes entailed in the first place a more thorough-going heliocentrism than that of Copernicus or Brahe. These two astronomers, following Ptolemy, had referred all motions to the mean Sun rather than the true Sun. Thus, in Copernicus, the lines of apsides of all the planets pass through the mean Sun ( $O$ in Fig. 7); i.e., through the center of the Earth's orbit. Kepler insisted that the lines of apsides all should pass through the body of the Sun itself. This change of hypothesis alters slightly the direction of the line of apsides, as well as the eccentricity.

In his treatment of the inequality in Mars' motion about the Sun, Kepler returned to the principle of equant motion. The outer planets, such as Saturn, traveled more slowly than the inner planets. It was not unreasonable to suppose,

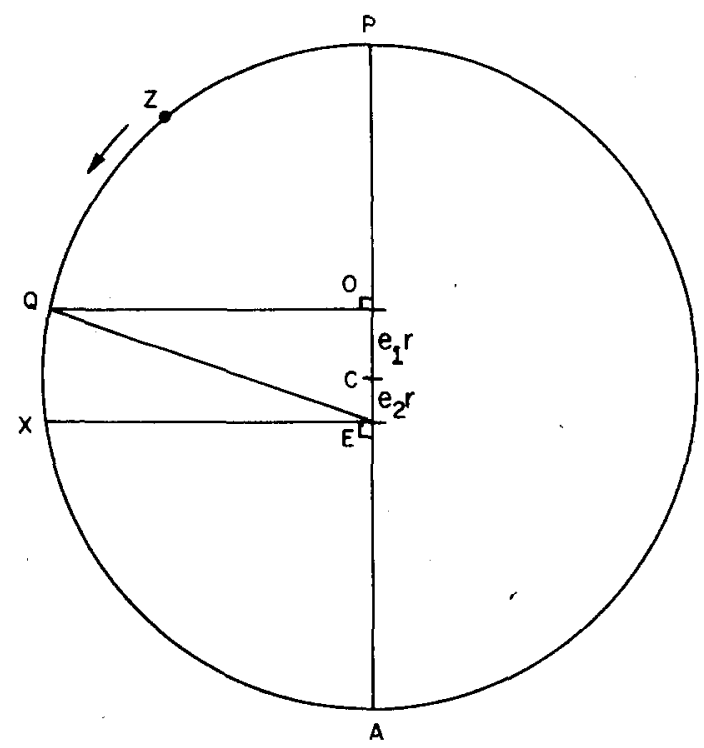

Fig. 10. Kepler's vicarious theory for the motion of Mars: an eccentric circle with an equant. The true Sun is at $O$, the center of the orbital circle at $C$, and the equant point at $E$.

then, that the speed of a given planet does physically vary with the planet's distance from the Sun-the planet traveling more slowly when farther from the Sun and more rapidly when nearer. The principle of equant motion directly addressed this variation in speed. In the nearly equivalent Copernican model, the physical variation in speed was hidden behind the guise of uniform circular motion.

Ptolemy had bisected the eccentricity of the superior planets without explicit justification and Copernicus had followed him. Brahe and Longomontanus had favored a 5:3 division. Kepler was unwilling to assume any a priori division, but sought to determine $e_{1}$ and $e_{2}$ (Fig. 10) directly from observation. This problem-to determine $e_{1}, e_{2}$, and the direction of the line of apsides-was more difficult than Ptolemy's version of the problem, in which $e_{1}$ and $e_{2}$ were assumed to be equal. Kepler's more general problem required the use of four oppositions rather than three. And while Ptolemy had used a clever method of iterations, in which successive corrections to an approximate solution were calculated, Kepler had to proceed more nearly by trial and error. It is in the description of this procecure that Kepler makes his famous remark, that if the reader finds the discussion tedious and difficult, he should pity the author, who had to perform the same lengthy calculation 70 times before arriving at the answer. ${ }^{18}$ The calculations were based on the oppositions of $1587,1591,1593$, and 1595 . Assuming that Mars moves on a circle in accordance with the law of the equant, Kepler found that the angular position of the planet was correctly reproduced at each of the four oppositions if

$$
\begin{aligned}
& e_{1}=0.11332, \\
& e_{2}=0.07232,
\end{aligned}
$$

while the total eccentricity $e_{1}+e_{2}$ came to 0.18564 . These values were not very different from those adopted by Brahe and Longomontanus. Indeed $e_{1} / e_{2} \approx 4.7 / 3$, while Brahe and Longomontanus had $e_{1} / e_{2}=\frac{5}{3}$. When Kepler checked the new theory against the 12 oppositions that fell between 1580 and 1604 , he found that in only four cases was the 


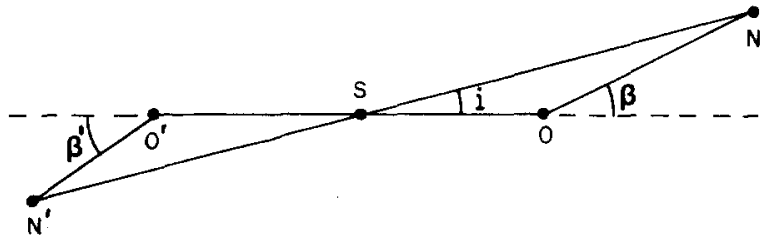

Fig. 11. Observation of the latitude $\beta$ of Mars while the planet is in opposition to the Sun at the northern $N$ or southern $N^{\prime}$ limit of its orbit.

discrepancy more than 1 min of arc, the worst being but $2^{\prime} 12^{\prime \prime}$. Discrepancies of this size could be attributed to the finite angular diameter of the planet and to the fact that the planet's parallax and the correction for atmospheric refraction were not known with great precision. ${ }^{19}$ It followed from the excellent agreement between the model and the 12 oppositions that, if the same procedure were used to derive $e_{1}$ and $e_{2}$ from a different set of four oppositions, very nearly the same result would be obtained. This appeared to be strong evidence for the validity of the theory.

And yet this theory that had cost so much labor, and that was so well confirmed by the dozen oppositions, was completely false. Kepler demonstrated the falsity of the vicarious hypothesis most directly by an investigation of Mars' latitudes while in opposition. (A planet's latitude is its angular distance above or below the plane of the Earth's orbit.) Especially useful were oppositions near the northern and southern limits of the planet's orbit. Refer to Fig. 11. Let $O O^{\prime}$ represent the plane of the Earth's orbit seen from the edge. Let $N N^{\prime}$ represent the plane of the orbit of Mars. Both planes pass through the Sun $S$. The inclination $i$ of the two planes Kepler had already determined. Consider an opposition of Mars when the Earth is at $O$ and Mars is at the northern limit $N$ of its orbit. Thus $S, O$, and $N$ lie in a plane that is perpendicular to the plane of the Earth's orbit. The latitude $\beta$ of the planet is found by observation. The distance $S O$ is given by the already completed theory of the Earth's motion. Thus, in triangle $S O N$, the angles at $S$ and $O$ are known, and side $S O$ is known, so side $S N$ may be calculated.

Similarly, consider a second opposition of Mars located $180^{\circ}$ further around in longitude, when the Earth is at $O^{\prime}$ and Mars is at the southern limit $N^{\prime}$ of its orbit. A measurement of the planet's latitude $\beta^{\prime}$ permits the calculation of $S N^{\prime}$. Then it is possible to compare $S N$ with $S N^{\prime}$. The difference between $S N$ and $S N^{\prime}$ leads directly to a value for the distance between the Sun and the midpoint of $N N^{\prime}$. Hence a value for $e_{1}$.

The results depended on the details of the adopted theory of the motion of the Earth, which affected the distances $S O$ and $S O^{\prime}$, but Kepler concluded that

$$
0.08000 \leqslant e_{1} \leqslant 0.09943 \text {, }
$$

while the vicarious hypothesis, so well supported by the dozen oppositions, required $e_{1}=0.11332$. Two methods of determining $e_{1}$ led to two different results. The theory was false.

Now, the total eccentricity in the vicarious hypothesis was 0.18564 . Half this was 0.09282 , a value that fell near the middle of the range of possible values for $e_{1}$ determined by the latitudes. Thus it appeared that a bisection of the eccentricity might be justified after all. Indeed, Kepler conjectured that Ptolemy must have settled on a bisection after experiencing similar difficulties with the latitudes.

A model with a bisected eccentricity (with $e_{\mathrm{P}}=0.09282$, half the total eccentricity in the vicarious hypothesis), will reproduce the longitudes of the oppositions located near aphelion or perihelion, as well as those located $\pm 90^{\circ}$ from these points. But when Kepler examined oppositions in the octants $\left( \pm 45^{\circ}\right.$ from either aphelion or perihelion) he found that the bisection model disagreed by 8 or 9 min of arc with the observations of Tycho Brahe. As Kepler remarks, Ptolemy did not claim to have observed with precision better than $10 \mathrm{~min}$, or a sixth of a degree. The uncertainty in Ptolemy's observations, therefore, exceeded the error in the model. At this point, Kepler makes his famous remark that, as divine Providence has accorded a conscientious observer in Tycho Brahe, whose observations have made apparent the 8-min error in the bisection model, it is proper to recognize and to honor this act of God, by searching into the true form of the celestial movements. These $8 \mathrm{~min}$ required the reform of all astronomy. ${ }^{20}$

\section{FIRST-ORDER ANALYSIS}

How well do the historically important theories of planetary motion correspond to the facts? Why does one method of determining the parameters lead to a 5:3 division of the eccentricity and another method lead to a bisection? The simple geometrical arguments of this and Sec. IV will resolve these questions.

\section{A. Simple eccentric circle}

If the simple eccentric circle [Hipparchos' solar theory of Fig. 3 or the intermediate form of the ancient planetary theory of Fig. 4(b) ] is to agree well with Kepler motion, the line $O C$ through the Earth and the center of the circular orbit should coincide with the major axis of the Keplerian ellipse. How big should the Hipparchian eccentricity $e_{\mathrm{H}}$ be? Let us start the model Sun at perigee $P$ at the right moment. By hypothesis, the Sun runs around the circle at uniform speed, completing its orbit in a year, whose length we denote $T$. It will reach apogee $A$ exactly half a year later, when it will again coincide with the real Sun. We may fix $e_{\mathbf{H}}$ by requiring that the model Sun also reach quadrature $Q$ at the correct instant. At $Q$, the Sun has moved through $90^{\circ}$ of the ecliptic as viewed from the Earth $O$.

Since the Sun moves uniformly on its circle, the time it requires to travel from $P$ to $Y$ is exactly one-fourth of a year, $T / 4$. Thus the time from perigee to quadrature is less than $T / 4$ by the time corresponding to the short arc $Q Y$. If the eccentricity $e_{\mathrm{H}}$ is much less than unity, $Q Y$ is approximately equal to $e_{\mathrm{H}} r$. Arc $P Q$ is then approximately $2 \pi r /$ $4-e_{\mathrm{H}} r$. This distance, divided by the Sun's speed $2 \pi r / T$, gives the time from perigee to quadrature in Hipparchos' model

$$
T_{P Q}=T / 4-e_{\mathrm{H}} T / 2 \pi \quad \text { (eccentric circle). }
$$

Let us now calculate the corresponding expression for the time between perigee and quadrature according to the rules of Kepler motion. Refer to Fig. 2. Elapsed time is proportional to the area swept out by the radius vector. The constant of proportionality is just the areal velocity $\pi a b / T$, the area of the ellipse divided by the length of the year. As the Sun moves through the $90^{\circ}$ from $P$ to $Q$, it sweeps out area $O P Q O$, which is smaller than one-quarter of the ellipse by 
the shaded area $O Q Y C O$. If the eccentricity $e$ is much less than unity, the shaded area is approximately rectangular and its area is eab. Thus the area swept out by the Sun's radius vector as the Sun moves from $P$ to $Q$ is approximately $\pi a b / 4-e a b$. This expression, divided by the areal velocity $\pi a b / T$, gives the time from perigee to quadrature in Kepler motion

$$
T_{P Q}=T / 4-e T / \pi \quad \text { (Kepler motion). }
$$

Comparison of Eqs. (1) and (2) reveals that Hipparchos' model will agree with the facts of planetary motion if $e_{\mathrm{H}}=2 \mathrm{e}$. That is, the Hipparchian eccentricity must be twice the eccentricity of the elliptical orbit. ${ }^{21}$

A few remarks about this result may be in order. The zero-eccentricity model was based on three hypotheses: (1) a circular orbit; (2) uniform motion; and (3) the centrality of the Earth. We know today that all three assumptions are wrong: (1) the orbit is an ellipse, (2) on which the Sun's speed varies and (3) the Earth is at one focus of the ellipse and not at the center. However, the elliptical nature of the orbit is of little consequence in comparison with the other two effects - the physical variation in the Sun's speed and the off-centeredness of the Earth. The greatest distance of the Sun from the center of its orbit is the semimajor axis $a$ of the ellipse. The Sun's least distance from the center is the semiminor axis $b$. Now, $b=a\left(1-e^{2}\right)^{1 / 2} \approx a\left(1-\frac{1}{2} e^{2}\right)$, so the departure from circularity is only of the second order in the eccentricity. On the other hand, effects (2) and (3) are first order. For an orbit of small eccentricity like the Sun's, it is nearly irrelevant that the orbit is an ellipse.

It happens that two important effects, the law of areas and the off-centeredness of the Earth, contribute equally to the nonuniformity of the Sun's angular speed around the Earth. Therefore, if we want to produce the whole effect from off-centeredness alone, we must make the off-centeredness twice as large. Thus we require $e_{\mathrm{H}}=2 e$ and not $e_{\mathrm{H}}=e$.

With $e_{\mathrm{H}}$ properly chosen, Hipparchos' model represents the angular motion of the Sun very well: The model Sun reaches four points of the ecliptic (perigee, apogee, and both quadratures) at exactly the right moments. (See Sec. V B below for a detailed discussion of the model's performance.)

If Hipparchos' model represents the Sun's angular position with good accuracy, it is much less satisfactory regarding the distance: The model predicts a variation in distance of $2 e$ above and below the mean, just twice the actual variation. This theoretical discrepancy, however, has few observable consequences. One might hope to measure the angular size of the Sun at apogee and at perigee, from which one could in principle calculate the actual variation in distance. Ptolemy tried to do this, as he says in Book V of the Almagest, but failed to find a perceptible change in the Sun's size. And no wonder-the measurements could only be made near sunrise or sunset, when the Sun's brightness is diminished to a tolerable level, but also when its shape is subject to the greatest refractive distortion; and anyway, the variation in angular diameter scarcely exceeds a minute of arc. Before the advent of telescopes and photography, no direct observation of the Sun could possibly have led to the conclusion that the solar eccentricity was too large by twice.

The eccentric circle was the standard theory of the Sun from the time of Hipparchos until the publication of Kepler's Astronomia nova. There were occasional modifi- cations of the numerical parameters, but the model itself could not be faulted. Even Kepler did not discover the model's defective treatment of the distances through direct observation of the Sun. Rather, the twofold exaggeration of the variation in the Earth-Sun distance was discovered because of its consequences for the apparent positions of the other planets in Kepler's reformed version of the heliocentric theory.

\section{B. Eccentric circle with equant point}

If the equant model is to represent satisfactorily the facts of Kepler motion, the line of apsides must coincide with the major axis of the Kepler ellipse. The question is, then, how the eccentricities $e_{1}$ and $e_{2}$ should be fixed. Let us establish a condition on the eccentricities by requiring, as before, that the time from perigee to quadrature be correct.

In Fig. 10, the Earth (or Sun, in a heliocentric model) is at $O$, the center of the deferent is at $C$, and the equant is at $E$. We seek an expression for the time of travel from perigee $P$ to quadrature $Q$. In equant motion, the time of travel is proportional to the angle subtended by the path at the equant. The constant of proportionality is just the constant angular speed $2 \pi / T$. Then, since $P E X$ is a right angle, the time from $P$ to $X$ is exactly one-fourth of a period, or $T / 4$. The time from $P$ to $Q$ is shorter than $T / 4$ by the time corresponding to angle $Q E X$. If the eccentricities $e_{1}$ and $e_{2}$ are much less than unity, $Q E X$ is a small angle and $Q E X$ $\approx\left(e_{1} r+e_{2} r\right) / r=e_{1}+e_{2}$. Thus angle $P E Q$ is $\pi /$ $2-\left(e_{2}+e_{2}\right)$. Division by the angular speed $2 \pi / T$ yields the time from perigee to quadrature in the equant model,

$$
T_{P Q}=T / 4-(T / 2 \pi)\left(e_{1}+e_{2}\right) \quad \text { (equant). }
$$

Comparing Eqs. (2) and (3), we see that motion with an equant will correctly represent the time from perigee to quadrature if $e_{1}+e_{2}=2 e$ : The sum of the eccentricity of the Earth and the eccentricity of the equant should be twice the elliptical eccentricity of the Kepler orbit.

It follows that the equant point $E$ coincides with the empty focus $F$ of the Keplerian orbit. In fact, it may be shown that, to a very good approximation, the empty focus behaves as an equant point. Mars does not move at uniform angular speed about the Sun. But if an observer could stand at the empty focus of the Martian orbit, he would indeed observe the planet move very nearly at constant angular speed. Ptolemy's introduction of the equant point into astronomy represented a profound insight into the nature of planetary motion.

\section{SECOND-ORDER ANALYSIS OF THE EQUANT MODEL}

Most of the historically important planetary models may be regarded as special cases of Kepler's own vicarious hypothesis, illustrated in Fig. 10. If $e_{2}=0$, the equant point $E$ coincides with the center of the circle and we obtain the solar theory of Hipparchos (Fig. 3). If we bisect the total eccentricity so that $e_{1}=e_{2}$, we have the planetary model favored by Ptolemy. If we put $e_{1}=\frac{5}{3} e_{2}$, we have a Ptolemaic form of the Copernican model used by Brahe and Longomontanus. And, as we have seen, Kepler experimented with two different divisions of the total eccentricity.

In Sec. III, we found that the equant model gives the correct time from perihelion to quadrature as long as the total eccentricity is $2 e$ : The division of the eccentricity is 
irrelevant. To determine the best division, we shall examine several other conditions that can be imposed on the vicarious hypothesis. In each case, we will calculate a simple expression for a time interval or an angle according to the vicarious hypothesis and compare this with a corresponding expression derived from Kepler's laws. All these expressions will be correct order $e^{2}$ : Finding the best division of the eccentricity, as opposed to determing the total eccentricity, inevitably requires the analysis of second-order effects. Throughout, we assume that the line of apsides $A P$ coincides with the major axis of the planet's true elliptical orbit. The planet's orbital period is denoted $T$. For simplicity of notation, we take the radius of the circular orbit (in the vicarious hypothesis) and the semimajor axis of the elliptical orbit (in the modern theory) to be unity.

\section{A. Condition I: Correct time from perihelion to quadrature}

By the comparison of Eqs. (2) and (3) we had

$$
e_{1}+e_{2}=2 e \quad \text { (condition } \mathrm{I} \text { ). }
$$

If condition I is satisfied, the vicarious hypothesis will put the planet in quadratures at the right times. Although condition I contains terms only of the first order in the eccentricities, it is in fact correct through the second order. The largest neglected terms are of order $e^{3.22}$
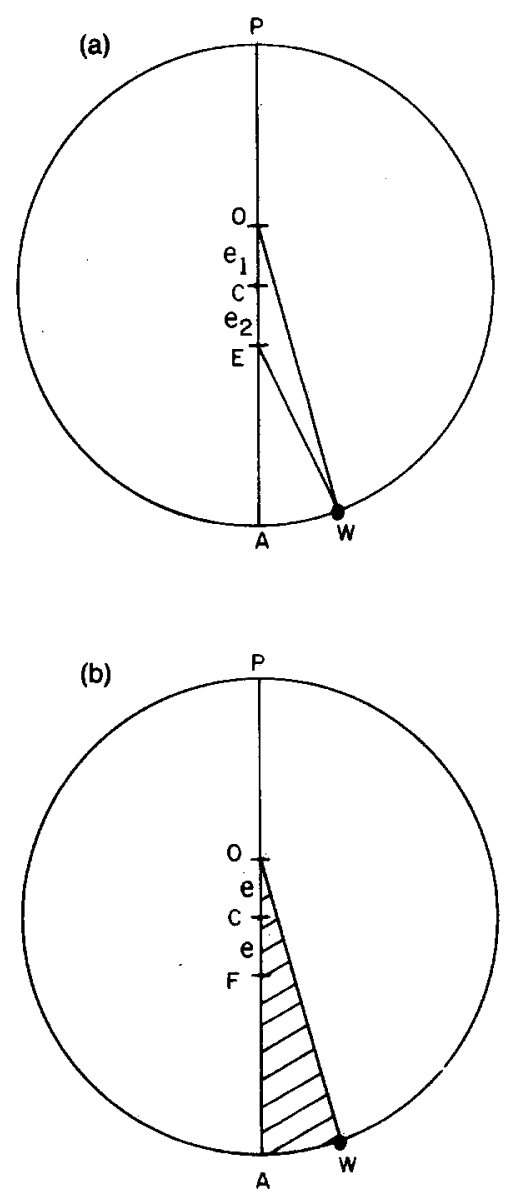

Fig. 12. Calculation of a planet's apparent angular speed at aphelion in an equant model (a) and according to Kepler's laws (b).

\section{B. Condition II: Correct angular speed at aphelion}

A second constraint one might choose to impose on the vicarious hypothesis is that it represents correctly the planet's angular speed in the apsides. Consider, first, the case of the aphelion. Let the planet, near aphelion $A$ in Fig. 12(a), travel $\operatorname{arc} A W$, which subtends angle $A E W$ at the equant. If this angle is small we have

$$
\Varangle A E W=A W / A E=A W /\left(1-e_{2}\right) \text {. }
$$

Then, by the property of the equant, the time required for the planet to run from $A$ to $W$ is

$$
T_{A W}=(T / 2 \pi)\left[A W /\left(1-e_{2}\right)\right] \text {. }
$$

In this same time, an imaginary observer on the Sun would see the planet travel through angle $A O W$. If the time interval is short, the angle is small and may be written

$$
\Varangle A O W=A W / A O=A W /\left(1+e_{1}\right) \text {. }
$$

Dividing the angle (5) through which the planet moves by the time interval (4) in which the motion occurs, we get

$$
\begin{aligned}
\Omega_{A} & =A O W / T_{A W} \\
& =(2 \pi / T)\left[\left(1-e_{2}\right) /\left(1+e_{1}\right)\right]
\end{aligned}
$$

(vicarious hypothesis),

which is the angular speed of the planet about the Sun at aphelion, according to the vicarious hypothesis.

Now we calculate the true angular speed at aphelion, according to Kepler's laws. Again, let the planet run arc $A W$, as in Fig. 12(b), while its radius vector sweeps out the area $O A W O$. As the arc is small, $O A W O$ is a triangle:

$$
\text { Area } O A W O=\frac{1}{2}(A W)(O A)=\frac{1}{2} A W(1+e) .
$$

Then, since the time of travel is proportional to the area swept out by the radius vector, the time the planet requires to go from $A$ to $W$ is

$$
T_{A W}=(T / \pi a b)(A W / 2)(1+e) .
$$

As before, we put $a=1$; the semiminor axis is then $b=\left(1-e^{2}\right)^{1 / 2}$. So the time of travel becomes

$$
T_{A W}=(T / 2 \pi)\left[A W(1+e) /\left(1-e^{2}\right)^{1 / 2}\right] .
$$

An imaginary observer on the Sun would see the planet move in this time through angle $A O W$ :

$$
\Varangle A O W=A W / O A=A W /(1+e) .
$$

The true angular speed of the planet about the Sun at aphelion is obtained by dividing the angle ( 8 ) by the time interval (7):

$$
\begin{aligned}
\Omega_{A} & =\Varangle A O W / T_{A W} \\
& =(2 \pi / T)\left(1-e^{2}\right)^{1 / 2} /(1+e)^{2}
\end{aligned}
$$

(Kepler motion).

Evidently, the vicarious hypothesis will give the correct angular speed at aphelion if the two expressions (6) and (9) for $\Omega_{A}$ are the same, that is, if

$$
\left(1-e_{2}\right) /\left(1+e_{1}\right)=\left(1-e^{2}\right)^{1 / 2} /(1+e)^{2} \text {. }
$$

This condition is exact; however, it is instructive to expand the right-hand side of the equation. Multiplication by $\left(1+e_{1}\right)$ and suppression of all terms of cubic order or higher in the eccentricities (i.e., terms like $e^{3}$ or $e_{1} e^{2}$ ) gives

$$
e_{1}+e_{2}=2 e+2 e e_{1}-\frac{5}{2} e^{2} \quad \text { (condition II). }
$$




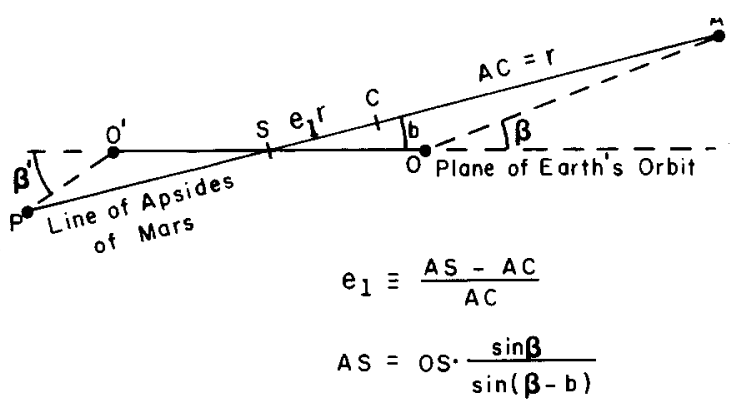

Fig. 13. Observation of a planet's geocentric latitude $\beta$ during an opposition to the Sun on the line of apsides.

\section{Condition III: Correct angular speed at perihelion}

A similar analysis may be applied to an arc near perihelion. The vicarious hypothesis will correctly represent the angular speed of the planet about the Sun at perihelion if

$$
\left(1+e_{2}\right) /\left(1-e_{1}\right)=\left(1-e^{2}\right)^{1 / 2} /(1-e)^{2} .
$$

Expansion to order $e^{2}$ gives

$$
e_{1}+e_{2}=2 e-2 e e_{1}+\frac{5}{2} e^{2} \quad \text { (condition III). }
$$

\section{Condition IV: Correct apsidal latitudes at opposition}

In the vicarious hypothesis, the motion of a planet about the Sun appears irregular for two reasons. First, there is a purely optical effect associated with $e_{1}$. Second, there is a physical variation in speed associated with $e_{2}$. Conditions I-III, based on the angular motion of the planet as observed from the Sun, naturally involves both the optical effect and the physical one, and so the mathematical relations contain $e_{1}$ as well as $e_{2}$. One might ask whether any sort of observation will permit the isolation of $e_{1}$ : Are there situations in which the purely optical effect is manifested without complications due to the physical variation in speed?

In fact, there are several such situations. We mentioned above Kepler's use of Mars' latitudes in opposition at the limits. Perhaps even simpler are the considerations involving the planet's latitude when it happens to be in opposition near one of its apsides. In Fig. 13 line $O O^{\prime}$ represents the plane of the Earth's orbit seen edge-on. $P A$ is Mars' line of apsides, with center $C$ midway between $P$ and $A$. These two lines intersect at the Sun $S$, where they form angle $b$.

Let Mars be at aphelion $A$ and let the Earth be at $O$, at the same heliocentric longitude, so that Mars is in opposition to the Sun. Now $O S$ and $O ' S$ are given by a theory of the

Table II. Divisions of the eccentricity resulting from various pairs of conditions.

\begin{tabular}{lllll}
\hline \hline Conditions & I & II & III & IV \\
\hline \multirow{2}{*}{ I } & - & $e_{1}=\frac{5}{4} e$ & $e_{1}=\frac{5}{4} e$ & $e_{1}=e$ \\
& $e_{2}=\frac{3}{4} e$ & $e_{2}=\frac{3}{4} e$ & $e_{2}=e$ \\
II & & $e_{1}=\frac{5}{4} e$ & $e_{1}=e$ \\
& - & $e_{2}=\frac{3}{4} e$ & $e_{2}=e$ \\
III & & - & $e_{1}=e$ \\
& & & $e_{2}=e$ \\
\hline \hline
\end{tabular}

Earth's motion. If $b$ and the radius $A C$ of Mars' circular orbit are known already, ${ }^{23}$ then a single observation of the planet's geocentric latitude $\beta$ while in opposition at aphelion will fix the eccentricity $e_{1}=S C / A C$. Or, if the radius is regarded as undetermined, two observations ( of $\beta$ and $\beta^{\prime}$ ) will fix the radius as well as $e_{1}$. The value of $e_{1}$ so obtained is a true measure of the distance between the Sun and the geometric center of the orbit, so the result will be ${ }^{24}$

$$
e_{1}=e \quad \text { (condition IV). }
$$

\section{E. The optimal division of the eccentricity}

We have obtained four different constraints by forcing the equant model to agree with various aspects of the true motion of the planets. The values an astronomer would calculate for $e_{1}$ and $e_{2}$ would therefore depend on the kind, and not only the quality, of data with which he worked.

If we combine condition IV with any one of the other three conditions, we are bound to obtain $e_{1}=e_{2}=e$. (See Table II.) The bisection of the eccentricity then looks rather good: This choice of parameters moves the planet from perihelion to quadrature in the right time and represents the apsidal latitudes correctly as well. The bisection therefore represents an excellent compromise, satisfying the first-order requirements in both angular motion and distance. (Of course, the bisection fails to produce the correct angular speed at perihelion and at aphelion-that is, the bisection fails to satisfy the second-order parts of conditions II and III.) The conditions we have derived apply strictly only to a heliocentric theory, but they apply with fair precision to a geometric theory like Ptolemy's if the eccentricity of the orbit corresponding to the epicycle is small compared to the eccentricity of the deferent. This situation obtains for every planet but Mercury. In this situation, any observational condition that requires correct representation of the distances (condition IV) will lead inevitably to a bisection. In his study of the superior planets, Ptolemy strove to represent correctly the widths of the retrograde arcs, especially of Mars. The varying widths of the retrograde arcs are partly due to an optical effect-the varying distance of the epicycle from the Earth as the epicycle moves around the eccentric deferent. Correct representation of the retrograde arcs therefore requires $e_{1}=e$. Again, in his study of Venus, Ptolemy was partly guided by an optical effect-the variation in the planet's greatest elongations from the Sun. This variation depends on the variation in the epicycle's distance from the Earth; its correct representation requires $e_{1}=e$. That Ptolemy settled on a bisection of the eccentricity is therefore hardly surprising.

If we ignore the purely optical condition (IV) and insist that the model represent very precisely the angular motion of the planet about the Sun, we are bound to obtain $e_{1}=5 e / 4$ and $e_{2}=3 e / 4$. Any pair of the three conditions I, II, III leads to this division. (See Table II.) The 5:3 division represents the angular motion very well. The planet moves from perihelion to quadrature in the right amount of time. The planet also has the correct angular speed at perihelion and at aphelion, so it will reach positions in the vicinity of both apsides at the right moments. In fact, at the precision acheivable with naked-eye observations even in Kepler's day, there is no perceptible error in the angular position anywhere around the orbit-as Kepler himself verified. However, the distances are incorrect in the first order: $e_{1}$ is 
Table III. Six models of planetary motion: Expansion in powers of the eccentricity.

\begin{tabular}{lcc}
\hline \hline & $\begin{array}{c}\text { True anomaly } \\
\theta(t)\end{array}$ & $\begin{array}{c}\text { Radius vector } \\
\rho(\theta)\end{array}$ \\
\hline $\begin{array}{l}\text { 1. Kepler motion } \\
\text { 2. Hipparchos' } \\
\text { solar theory } \\
e_{1}=2 e, \quad e_{2}=0\end{array}$ & $\omega t+2 e \sin \omega t+\frac{5}{4} e^{2} \sin 2 \omega t$ & $1-e \cos \theta-e^{2} \sin ^{2} \theta$ \\
3. Ptolemy's \\
planetary theory \\
$e_{1}=e, \quad e_{2}=e$ \\
$\begin{array}{l}\text { 4. Kepler's } \\
\text { vicarious theory } \\
e_{1}=\frac{5}{4} e, \quad e_{2}=\frac{3}{4} e\end{array}$ \\
$\begin{array}{l}\text { 5. Copernicus' } \\
\text { planetary theory } \\
a=\frac{1}{2} e, \quad b=\frac{3}{2} e\end{array}$ \\
$\begin{array}{l}\text { 6. Brahe's } \\
\text { theory of Mars } \\
a=\frac{3}{8} e, \quad b=\frac{13}{8} e\end{array}$ \\
\hline \hline
\end{tabular}

put equal to $\frac{5}{4} e$ rather than to $e$. Thus any effect depending on the distances (latitudes, widths of retrograde arcs, etc.) will be poorly represented-as Kepler discovered to his chagrin.

\section{APPENDIX: DEVELOPMENT IN POWERS OF THE ECCENTRICITY}

Comparison of the historically important models of planetary motion with the modern theory is particularly simple when the position of the moving body is developed in powers of the eccentricity. The expressions presented below are not new, but it may be convenient to have them grouped in one place.

\section{A. Kepler motion}

The equation for the elliptical orbit may be written

$$
\rho=a\left(1-e^{2}\right) /(1+e \cos \theta) \text {, }
$$

where $\rho$ is the planet's distance from the Sun and $\theta$ (the "true anomaly") is its angular distance from perihelion. As usual, $a$ represents the semimajor axis and $e$ the eccentricity.

The planet moves upon the ellipse so that the radius vector sweeps out equal areas in equal times. The constant areal velocity is just $\pi a b / T$, the area of the ellipse divided by the orbital period. Then, since the semiminor axis $b=a\left(1-e^{2}\right)^{1 / 2}$, the condition of constant areal velocity is

$$
\frac{\rho^{2}}{2} \frac{d \theta}{d t}=\frac{\pi a^{2}}{T}\left(1-e^{2}\right)^{1 / 2} \text {. }
$$

Equations (A1) and (A2) - the shape of the orbit and the speed law-completely determine the motion. For small e, Eq. (A1) may be expanded by means of the binomial theorem, with the result

$$
\rho(\theta)=a\left(1-e \cos \theta-e^{2} \sin ^{2} \theta+\cdots\right) .
$$

When this expression for $\rho(\theta)$ is substituted into Eq. (A2) and the resulting differential equation for $\theta(t)$ is expanded and integrated through order $e^{2}$, we obtain ${ }^{25}$

$$
\theta(t)=\omega t+2 e \sin \omega t+\frac{5}{4} e^{2} \sin 2 \omega t,
$$

where $\omega=2 \pi / T$. The expressions (A3) and (A4) for $\rho(\theta)$ and $\theta(t)$ are displayed in the first line of Table III. In the expression for the radius vector, the semimajor axis has been set equal to unity.

\section{B. Eccentric circle with equant}

In Fig. 14, the radius of the eccentric circle has been set equal to unity. Here, $e_{1}$ and $e_{2}$ denote the eccentricity of the Earth $O$ and of the equant $E$. The equation of the orbit in

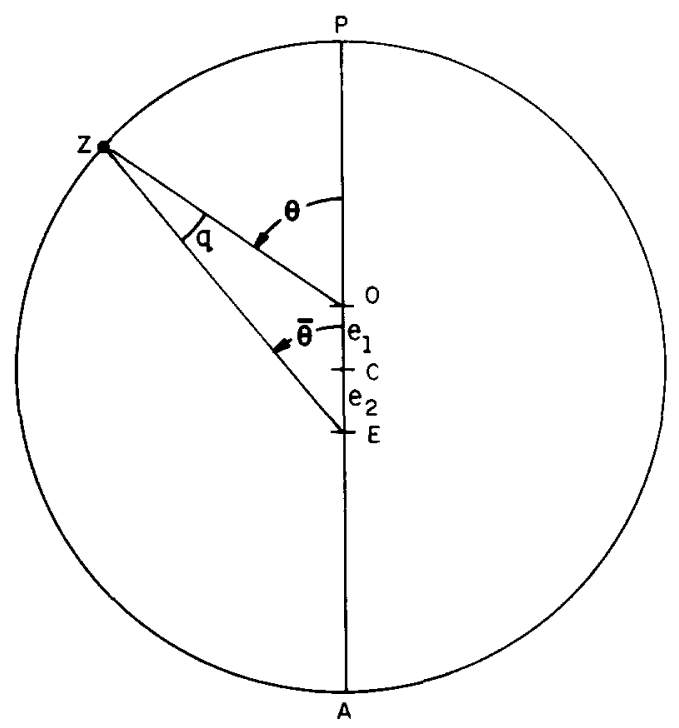

Fig. 14. Calculation of the equation of center $q$ and the radius vector $\rho$ in the eccentric with equant model. 
polar coordinates is that of a circle eccentric to the origin:

$$
\begin{aligned}
\rho & =-e_{1} \cos \theta+\left(1-e_{1}^{2} \sin ^{2} \theta\right)^{1 / 2} \\
& \approx 1-e_{1} \cos \theta-\frac{1}{2} e_{1}^{2} \sin ^{2} \theta .
\end{aligned}
$$

The problem at hand is to determine the true anomaly $\theta$ as a function of time. Now the mean anomaly $\bar{\theta}$, by the defining property of the equant, increases uniformly with time:

$$
\bar{\theta}=\omega t \text {. }
$$

In triangle $Z O E$, angle $q$ (the "equation of center") is equal to $\theta-\bar{\theta}$. Application of the law of sines gives

$$
\theta=\bar{\theta}+\sin ^{-1}\left\{\left[\left(e_{1}+e_{2}\right) / \rho\right] \sin \bar{\theta}\right\} .
$$

Equation (A5) may be substituted for $\rho$. Expansion to the second order in the eccentricities then yields

$$
\theta=\omega t+\left(e_{1}+e_{2}\right) \sin \omega t+\frac{1}{2} e_{1}\left(e_{1}+e_{2}\right) \sin 2 \omega t .
$$

Hipparchos' eccentric-circle theory of the Sun produces approximately the correct angular motion if the Hipparchian eccentricity is identified with twice the elliptical eccentricity. Thus, in Eqs. (A5) and (A6), we put $e_{1}=2 e$, $e_{2}=0$. The results are displayed in line 2 of Table II. Here, $\theta(t)$ is correct through order $e$, the first discrepancy being of order $e^{2}$. In the radius vector, however, the first discrepancy is of the first order in $e$, as already mentioned. The error in the angular position is $\frac{3}{4} e^{2} \sin 2 \omega t$, which reaches a maximum in the octants. For the Sun, $e=0.0167$, so the maximum error is less than a minute of arc, far below the precision of observation achieved in antiquity. The ancient solar theory did not, of course, actually achieve its full potential accuracy because of errors in the observations used to determine the numerical parameters of the model.

If in Eqs. (A5) and (A6) we put $e_{1}=e_{2}$, we have Ptolemy's model for the motion of a superior planet's epicycle about the Earth. The model represents with equal validity the motion of a planet about the Sun. As has been shown, good agreement with Kepler motion is obtained by putting $e_{1}=e_{2}=e$. See line 3 of Table III. Now $\rho$ is correct to first order in $e$. The error in $\theta$ is now only $-\frac{1}{4} e^{2} \sin 2 \omega t$, which reaches a maximum in the octants. Even for Mars, with its large (0.093) eccentricity, the maximum error is only 7 or $8 \mathrm{~min}$ of arc. Indeed, these are the famous 8 min of arc that caused Kepler to reject the Ptolemaic eccentric and equant model with bisected eccentricity.

If in Eqs. (A5) and (A6), we put $e_{1}=\frac{5}{4} e$ and $e_{2}=\frac{3}{4} e_{1}$, we have the optimal form of Kepler's vicarious hypothesis. See line 4 of Table III. The angular motion is now represented perfectly through order $e^{2}$. Residual errors, of order $e^{3}$, are too small to be detected with naked-eye instruments. However, there is now an error in the distance of order $e$. Thus Kepler found that the vicarious model represented Mars' angular motion perfectly adequately, but failed to give a proper account of the latitudes and the lengths of the retrograde arcs.

\section{Copernicus' theory of the superior planets}

Refer to Fig. 15, where $C$ is the center of the deferent circle whose radius has been set equal to unity; $E$, the effective equant point, is located at distance $a$ below $C$; thus angle $O E Z$ is equal to the mean anomaly $\bar{\theta}$, which increases uniformly with time: $\bar{\theta}=2 \pi t / T$.

In the trapezoid $E C D Z$, denote side $E Z$ by $d$. Now $d=1+Z G=H E$, and $H E=Z G=a \cos \bar{\theta}$. Thus

$$
d=1+2 a \cos \bar{\theta}
$$

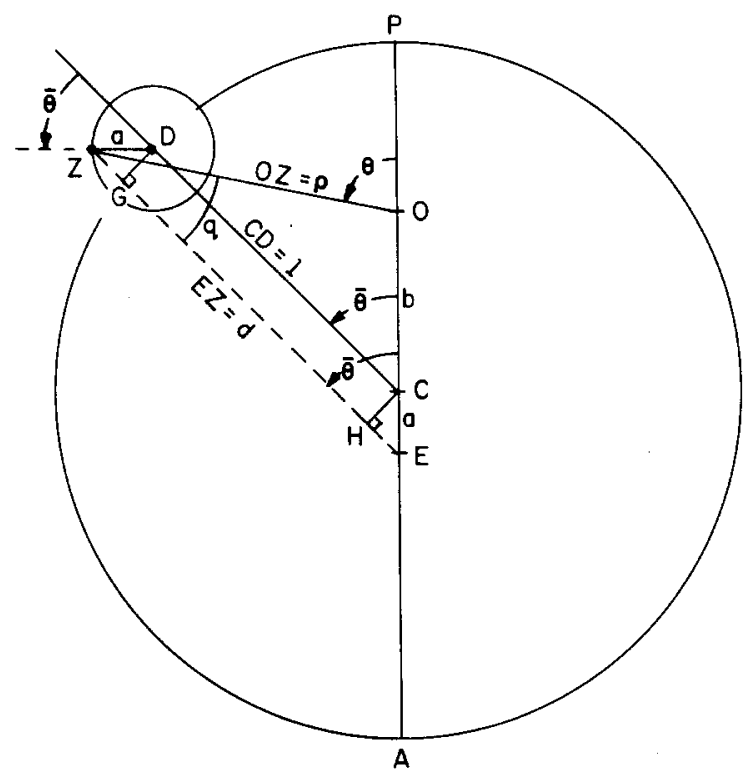

Fig. 15. Calculation of the equation of center $q$ and the radius vector $\rho$ in the Copernican model.

Application of the cosine law in triangle $E O Z$ gives

$$
\rho=\left[d^{2}+(a+b)^{2}-2(a+b) d \cos \bar{\theta}\right]^{1 / 2} \text {. }
$$

Upon substitution of the expression for $d$ and expansion to the second order in $a$ and $b$, we obtain

$$
\rho=1-(b-a) \cos \bar{\theta}+\frac{1}{2}(a+b)^{2} \sin ^{2} \bar{\theta} .
$$

In triangle $E Z O$, angle $q$ is the equation of center, i.e., the difference between the mean and the true anomaly: $q=\theta-\bar{\theta}=\theta-\omega t$. The law of sines yields

$$
\theta=\bar{\theta}+\sin ^{-1}\{[(a+b) / \rho] \sin \bar{\theta}\} \text {. }
$$

Substitution of Eq. (A7) for $\rho$ and reexpansion yields

$$
\theta=\omega t+(a+b) \sin \omega t+\frac{1}{2}\left(b^{2}-a^{2}\right) \sin 2 \omega t .
$$

It remains to express the radius vector $\rho$ directly in terms of $\theta$ rather than $\vec{\theta}$. We shall truncate the expansion at the second order in $a$ and $b$. Thus, in Eq. (A7) in the term involving $(a+b)^{2}, \bar{\theta}$ may simply be replaced by $\theta$. But, in the term involving $(b-a)$, we must put $\bar{\theta}=\theta-(a+b)$ $\sin \theta$ [from Eq. (A8) ]. Upon reexpansion, Eq. (A7) then becomes

$\rho=1-(b-a) \cos \theta+\left[\frac{1}{2}(a+b)^{2}+a^{2}-b^{2}\right] \sin ^{2} \theta$.

In Copernicus' version of this model, $b=3 a$. As demonstrated above, the model will agree well with the true angular motion of the planets if $a+b=2 e$. Thus we put $a=\frac{1}{2} e$ and $b=\frac{3}{2} e$ in Eqs. (A8) and (A9). The resulting forms of $\theta(t)$ and $\rho(\theta)$ are on line 5 of Table III. It is useful to compare Copernicus' model with the corresponding Ptolemaic model (line 3). The Copernican expression for $\theta(t)$ is indistinguishable from the Ptolemaic expresssion, through order $e^{2}$. This reflects the fact that Copernicus' model contains a hidden equant point and that Copernicus, following Ptolemy, effectively bisected the total eccentricity. Copernicus' speed rule is thus the same as Ptolemy's. ${ }^{26}$ The Ptolemaic model, however, gives a better treatment of the distances.

The second historically important version of the Copernican model is that of Brahe and Longomontanus. Here, 
$b=\frac{13}{3} a$ and, as before, the best agreement with Kepler motion results when $a+b=2 e$. Thus we obtain $a=\frac{3}{8} e$, $b=\frac{13}{8} e$. See line 6 of Table III. Brahe's model should be compared with Kepler's vicarious hypothesis (line 4), for both of these models adopt an effective 5:3 division of the total eccentricity. The two expressions for $\theta(t)$ in lines 4 and 6 are indistinguishable; and, in the radius vector, Brahe's theory has an error of order $e$, as does the vicarious hypothesis.

The dilemma confronted by Kepler may be grasped by comparing the bisection of the eccentricity (line 3 ) with the 5:3 division (line 4). The bisection represents the distances properly in order $e$, but commits an error of order $e^{2}$ in the angular motion. The 5:3 division gets the angular motion right but commits an error of order $e$ in the distances. It is, of course, anachronistic to pose the dilemma in terms of series. Nevertheless, it was the impossibility of representing all aspects of the motion of Mars with a single version of the eccentric circle and equant model that moved Kepler to seek the true shape of the orbit.

\section{Kepler motion observed from the empty focus}

In the Keplerian ellipse of Fig. 16, the true anomaly $\theta(t)$ is given by Eq. (A4); and the radius vector $\rho(\theta)$, by Eq. (A3). We seek an expression for $\varphi(t)$, representing the motion of the planet as observed from the empty focus. By a property of the ellipse, $h=2 a-\rho$, or if we put the semimajor axis equal to unity, $h=2-\rho$. Application of the law of sines to triangle $O Z F$ gives

$$
\sin (\theta-\varphi)=2 e \sin \theta / h=2 e \sin \theta /(2-\rho) .
$$

Substitution of the already derived expressions for $\rho(\theta)$ and $\theta(t)$ [Eqs. (A3) and (A4)] and expansion yields

$$
\varphi(t)=\omega t+0+\frac{1}{4} e^{2} \sin 2 \omega t .
$$

There is no term linear in $e$. Thus, through order $e, \varphi$ develops simply as $\omega t$ : To order e, the empty focus behaves as an equant point. An observer standing on the Sun would not see Mars move at uniform angular speed. From Eq. (A4), the planet's greatest angular distance (in radians) from its

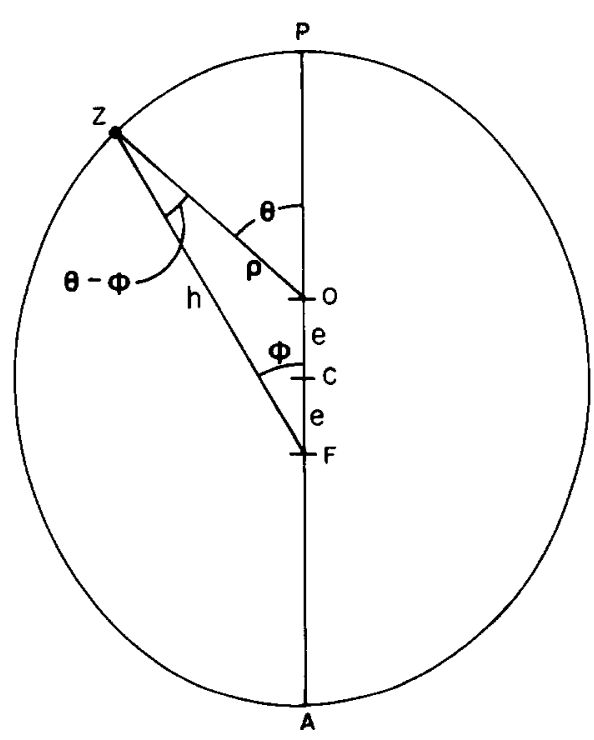

Fig. 16. The motion viewed from the empty focus $F$ of a planet's elliptical orbit. uniformly moving mean position is roughly $2 e$. For Mars $(e=0.093)$, this amounts to $10.7^{\circ}-$ a very considerable departure from uniform angular motion. For an observer at Mars' empty focus, however, the planet's greatest angular departure from its uniformly moving mean position is, from Eq. (A10), $\frac{1}{4} e^{2}$ (in radians), or about $0.1^{\circ}$. Thus, at the level of observational precision achievable in antiquity, the empty focus of the Keplerian ellipse is indistinguishable from an equant point.

'J. Kepler, in Johannes Kepler Gesammelte Werke, Vol. III, edited by M. Casper and W. von Dyck (Munich, 1937). There is a photoreproduction of the 1609 edition (Culture et Civilisation, Brussels, 1968); a German translation by M. Casper, Neue Astronomie (Oldenbourg, Munich, 1929); a French translation by J. Peyroux, Astronomie nouvelle ("chez le traducteur," 1979); and an English translation by W. Donahue is forthcoming from Cambridge U.P.

${ }^{2}$ For a brief account of the historical development of the Greek deferent and epicycle theory, and a discussion of the observational evidence that probably was of greatest importance in the evolution of the theory, see $\mathbf{J}$. Evans, Am. J. Phys. 52, 1080 (1984).

${ }^{3}$ Most of what we know of Hipparchos' contributions to planetary theory we owe to Ptolemy's brief references in the Almagest. The Greek text of the Almagest has been edited by J. L. Heiberg, Claudii Ptolemaei Opera quae exstant omnia (Teubner, Leipzig, 1898, 1903), Vol. I, 2 parts. Two English translations are available: Ptolemy's Almagest, G. J. Toomer, trans. (Duckworth, London, 1984) and The Almagest, R. C. Taliaferro, trans. in Great Books of the Western World (Encyclopaedia Britannica, Chicago, 1952), Vol. 16.

${ }^{4}$ The elliptical eccentricity $e$ of Mars' orbit was in Ptolemy's day about 0.09 . However, Ptolemy's figure of 0.10 for $e_{\mathrm{P}}$ is better than it might seem at first sight. As will be demonstrated in Sec. IV, Ptolemy's model works well if $e_{P}=e$, as long as the eccentricity of the Earth's orbit can be neglected. If the Earth's eccentricity is taken into account, the situation is more complicated, for this corresponds to an eccentricity in the epicycle. In the intermediate model, good agreement between model and motion results if the eccentricity of the deferent is put equal to the vector difference of the eccentricities (weighted by the radii of the orbits) of Mars and the Earth. This vector difference was much closer to 0.10 than to 0.09. In the full Ptolemaic model with equant point, no such simple correspondence can be stated; however, $e_{\mathbf{P}}=0.1$ was still a better value than 0.09 would have been. For a discussion of this subject, see $\mathrm{O}$. Neugebauer, $A$ History of Ancient Mathematical Astronomy (Springer-Verlag, Berlin, 1975), pp. 146-148.

${ }^{5}$ The discussion has been expressed in terms of circular orbits. The mean Sun is the center of the Earth's orbit. Since the Earth's circular orbit is slightly eccentric, the true Sun is not at $S$, but is slightly displaced from $S$. The opposition of Mars to the true Sun could occur as much as a day and a half before or after the mean opposition. Kepler was the first to introduce the use of true, rather than mean, oppositions.

In terms of a geocentric model, the mean Sun is a fictitious body that moves uniformly on a circle centered on the Earth. The true Sun, which moves uniformly on a circle eccentric to the Earth, may be as much as $2^{\circ}$ ahead of or behind the mean Sun.

${ }^{6}$ The derivation of the eccentricity and the longitude of the perigee from three mean oppositions is a difficult geometrical problem. Ptolemy could find no direct solution and was forced to adopt an ingenious method of iterations. Ptolemy's solution is a geometrical tour de force, and is, indeed, the most impressive piece of applied mathematics from the whole ancient period. For a detailed discussion of Ptolemy's technique, see O. Neugebauer, A History of Ancient Mathematical Astronomy (Springer-Verlag, New York, 1975), pp. 172-179.

${ }^{7}$ Copernicus, De revolutionibus orbium coelestium (Nuremberg, 1543). There are several modern reprints. Three English translations are available: On the Revolutions, edited by J. Dobrzycki, E. Rosen, trans. ( Johns Hopkins, Baltimore, 1978); On the Revolutions, A. M. Duncan, trans. (Barnes \& Noble, New York, 1976); On the Revolutions of the Heavenly Spheres, C. G. Wallis, trans., in Great Books of the Western World (Encyclopaedia Britannica, Chicago, 1952), Vol. 16. Copernicus alludes to Ptolemy's violation of the principle of uniform circular motion in the 
preface to De revolutionibus, but his most vigorous criticism of Ptolemy's practice is at the beginning of the discussion of the lunar theory. (Book IV, Chap. 2).

${ }^{8}$ Copernicus, who in the ancient fashion refers the motion of $Z$ to the turning line $C D$, says that $D Z$ and $C D$ turn at the same rate. If we wished to refer all motions to directions fixed in space, in modern fashion, we would say that $D Z$ turns twice as fast as does $C D$.

${ }^{9}$ Copernicus, Book V, Chap. 4. The flattened character of the orbit is easily shown by comparision of the planet's distance from the orbit center $M$ at $0^{\circ}$ and at $90^{\circ}$ of mean anomaly; i.e., at positions 1 and 3 in Fig. 6. In position 1 we have $M Z=C D-C M+D Z=r-a r+a r=r$. But in position 3 we have $M Z=\left[C D^{2}+(D Z+M C)^{2}\right]^{1 / 2}=r\left(1+4 a^{2}\right)^{1 / 2}$, which is somewhat greater than $r$.

${ }^{10}$ When Copernicus introduces his model for the motion of the three superior planets (Book V, Chap. 4), he does not explain how he arrived at these particular details. Nor does he ever mention the existence of a hidden equant point ( $E$ in Fig. 7). It is possible that Copernicus neglected to mention that his model had an effective equant point in order not to appear to follow Ptolemy in a principle that he, Copernicus, had rejected. However, Copernicus is usually quite open in discussing the relation between his theories and those of Ptolemy, whose practice he follows more often than not. It is more likely that Copernicus did not fully understand how nearly perfectly his model duplicated Ptolemy's-that he did not perceive that the angular motion was uniform with respect to point $E$. The earliest proof of the existence of an equant point in Copernicus' model is contained in a letter of the year 1595 from Michael Maestlin, professor of mathematics at Tübingen, to his former pupil, Kepler. See A. Grafton, Proc. Am. Philos. Soc. 117, 523 (1973). Kepler wrote back to his teacher, "Now at last I understand this business" about the equant.

How could Copernicus have arrived at his model without appreciating fully its near equivalence to Ptolemy's? The following considerations may have been foremost. First of all, the minor epicycle revolving at the same rate and in the same direction as the deferent was discussed and rejected by Ptolemy in Almagest III, 3 as an alternative version of the solar theory. The basic mechanism of the model was therefore at Copernicus' disposal.

In Ptolemy's discussion, the eccentricity of the deferent is zero; i.e., $b=0$ in Fig. 6. Copernicus could not, however, apply the $b=0$ form of the model to Mars: For, in this form, the model produces a more rapid motion of the planet at aphelion, and a slower motion at perihelion, which was the reverse of the natural order and of Ptolemaic practice. (With $b=0$ in Fig. 6, the mean Sun $O$ would be at $C$, and position 1 would be aphelion.) The introduction of an eccentricity $b$ of the deferent sufficed to turn position 1 into the perihelion, and so made the motion most rapid at perihelion, as required.

Putting $a+b=2 e_{\mathrm{p}}$ would make the equation of center at $90^{\circ}$ of mean anomaly virtually the same as in Ptolemy's model. [Thus, $O Z$ in Fig. 9(a) and $O Z$ in Fig. $9(\mathrm{~b})$ will be in virtually the same direction if $a+b=e_{1}+e_{2}$.] Finally, putting $b-a=e_{1}$ would preserve the same distance between $O$ and the center of the effective orbit as in Ptolemy-a condition clearly necessary for retrogradations of the correct width.

Nearly 200 years before Copernicus, Ibn al-Shātir of Damascus proposed to replace Ptolemy's equant by exactly the same mechanism. It is conceivable that Copernicus drew on an unknown Latin translation or synopsis of Ibn al-Shātir's work. However, no such connection has been demonstrated. One must therefore allow the possibility of independent discovery. See N. M. Swerdlow, Proc. Philos. Soc. Am. 117, 423 (1973).

${ }^{1}$ N. M. Swerdlow and O. Neugebauer, Mathematical Astronomy in Copernicus's De Revolutionibus (Springer, New York, 1984), p. 298.

${ }^{12}$ The 3 ' maximum difference between the equations of center of the two models for Mars presupposes the identifications $a=\frac{1}{2} e_{\mathrm{P}}$ and $b=\frac{3}{2} e_{\mathrm{P}}$. An even better agreement between the models can be obtained if the Copernican parameters $a$ and $b$ are slightly increased above these values. Let us compare the equations of center in the two models at $90^{\circ}$ of mean anomaly, i.e., when $\Varangle P E Z$ is a right angle. From Fig. 9(a), the equant model yields in this situation $\tan q=\left(e_{1}+e_{2}\right) /\left(1-e_{2}^{2}\right)^{1 / 2}$. From Fig. 9 (b), the Copernican model yields $\tan q=a+b$. The two models will yield exactly the same value for the equation of center $q$ in this situation if we put $a+b=\left(e_{1}+e_{2}\right) /\left(1-e_{2}^{2}\right)^{1 / 2} \simeq\left(e_{1}+e_{2}\right)\left(1+\frac{1}{2} e_{2}^{2}\right)$. For Ptolemy's model, in which $e_{1}=e_{2} \equiv e_{\mathrm{p}}$, the correspondence becomes $a+b=2 e_{\mathrm{p}}+e_{\mathrm{p}}^{3}$. Thus Copernicus' version of the correspondence $\left(a+b=2 e_{\mathrm{p}}\right)$ is perfectly fine through the second order in the eccentricities: The correction that must be applied to remove the $3^{\prime}$ difference in the quadrants is $e_{\mathbf{p}}^{3}$. For the case of Mars, this implies an increase in the total eccentricity $a+b$ of $0.5 \%$ over and above the value obtained from the sample correspondence $a+b=2 e_{\mathrm{p}}$. If this modified correspondence is adopted, the Copernican model will not disagree with the equant model by as much as a minute of arc anywhere around the orbit. It appears that Kepler was the first to realize that the total Copernican eccentricity must be increased very slightly above the total eccentricity in the equant model. (Ref. 1, I, iv.) When Kepler passes back and forth between the equant model and the minor epicycle model, he habitually takes into account the slight increase in the Copernican eccentricity required for perfect correspondence.

${ }^{13}$ Ptolemy expressed his eccentricities as base- 60 fractions. When they are expressed in base-10, as in Table I, they may appear somewhat overprecise. Copernicus expressed the eccentricities as base-10 fractions, using a denominator of 10000 .

${ }^{14}$ There are some problems connected with Copernicus' derivations of the eccentricities of the superior planets. In spite of inconsistent procedures, and occasional mistakes of computation, Copernicus succeeds in confirming Ptolemy's values for the total eccentricities of Jupiter and Saturn from observations that he, Copernicus, made himself. It appears that in this matter Copernicus deliberately sought agreement with Ptolemy. Copernicus' minor reduction in the eccentricity $b$ of the Martian deferent is not very clearly explained. This adjustment was not a direct consequence of any attempt to fit the observed longitudes of the mean oppositions. Rather, it appears that Copernicus made the adjustment because his calculated motion of the Martian line of apsides implied that the distance between the mean Sun and the center of Mars' deferent had slightly decreased since Ptolemy's day. See the discussion by Swerdlow and Neugebauer, Ref. 11, pp. 323-369.

${ }^{15}$ Christen Sørensen (1562-1647). Son of a poor farmer from the village of Lomborg in the northwest of Jutland, he acquired an education through great personal determination. From 1589 he was Brahe's assistant at Hven, and when Brahe left Denmark S $\phi$ rensen followed him. As was customary in his day, he Latinized his name and the name of his native village, styling himself Christianus Severinus Logomontanus. He is best known today for his astronomical treatise, Astronomica Danica, of which there were three printings between 1622 and 1640 . Interestingly, although Longomontanus worked closely with Kepler in Prague, he did not fully accept Kepler's discoveries. He followed Kepler in using the oppositions of the superior planets to the true, rather than the mean, Sun. But he did not accept the elliptical orbit. For biographical details, see J. L. E. Dreyer, Tyco Brahe: A Picture of Scientific Life and Work in the Sixteenth Century (Black, Edinburgh, 1890), p. $126 \mathrm{ff}$. For a survey of the Astronomica Danica, see J. B. J. Delambre, Histoire de l'astronomie moderne (Paris, 1821), Vol. I, pp. 262-287.

${ }^{16}$ In dicussing his own work, Kepler provides a good deal of detail on the state of the theory of Mars as he received it from Brahe and Longomontanus. The numerical values of $a$ and $b$, as well as the equivalent values of $e_{1}$ and $e_{2}$ that had been adopted by Brahe are mentioned Astronomia nova, I, iv (p. 15 in the edition of 1609 , but mistakenly printed 14 ). The numerical parameters of the model are again mentioned in at II, vii ( $p$. 53), where Severinus (Longomontanus) is explicitly given credit. This working theory of Brahe and Longomontanus was discussed briefly by Small (Ref. 17, p. 154).

${ }^{17}$ Two studies of Kepler's Astronomia nova are of great value. R. Small, $A n$ Account of the Astronomical Discoveries of Kepler (London, 1804; reprint edition: University of Wisconsin, Madison, 1963). Alexandre Koyré, La révolution astronomique (Hermann, Paris, 1961); English translation by R. E. W. Maddison, The Astronomical Revolution (Hermann, Paris, 1973).

${ }^{18}$ Kepler, Ref. 1, II, xvi (p. 95). Kepler's request for pity is often quoted sympathetically. But Delambre, who estimated that these 70 calculations would run to a mere 200 manuscript pages, repeated some of them and found them "not so terrible." "One has more calculations to make today to determine the elliptical orbit of a comet." J. B. J. Delambre, Histoire del'astronomie moderne (Paris, 1821), Vol. I, p. 417. Delambre adds that "Kepler was sustained by the desire to be right against Tycho, Copernicus, Ptolemy, and all the astronomers of the universe; he tasted 
this satisfaction, and I do not believe that he was so worthy of pity when he made all these calculations."

${ }^{19}$ Kepler, Ref. 1, II, xix (p. 109).

${ }^{20}$ Kepler, Ref. 1, II, xix (pp. 113-114).

${ }^{21}$ This result is familiar to historians of astronomy; however, its derivation is usually much more complicated than the derivation given here. See, for example, A. Aaboe, Centaurus 5, 212 (1958).

${ }^{22}$ The truth of this assertion is easily demonstrated. Our approximations consisted of replacing the tangent of a small angle by the angle itself, and in identifying $O Q$ or $E X$ with the radius of the circular orbit (in Fig. 10) or with the semiminor axis of the elliptical orbit (in Fig. 2). The Taylor series expansion of $\tan \theta$ is $\theta+\theta^{3} / 3+\cdots$, so that there is no term in $\theta^{2}$, which disposes of the first approximation. At point $Y$ in Fig. 2 the tangent to the ellipse is parallel to the major axis; thus, near $Y$, the ellipse may be approximated by a parabola with horizontal axis. This means that the distance $O Q$ can be written as $b$ minus a term in $e^{2} b$ : There is no term linear in $e$. Indeed it is easy to show that $O Q=b\left(1-\frac{1}{2} e^{2}+\cdots\right)$. Then, when we find the area of zone $O Q Y C O$, we will essentially multiply by a factor $e a$, which will give us order $e^{3}$ as the first correction to our previous result for this area. A similar argument can be applied to the approximation $E Q=r$ in the case of the circular orbit of Fig. 10 .

${ }^{23}$ Angle $b$ is not, of course, the same as the inclination $i$ of the planet's orbit to the plane of the ecliptic unless the orbit's line of apsides happens to be $90^{\circ}$ away from the line of nodes. Let $\omega$ denote the argument of perihelion (i.e., the angular distance, measured in the plane of the orbit, from the ascending node to the perihelion). Then $\sin b=\sin i \sin \omega$.

${ }^{24}$ Conditions I-III, based on the apparent angular motion of the planet observed from the Sun, involve both $e_{1}$ and $e_{2}$. Condition IV, based on a purely optical effect-the apparent geocentric latitude of the planetdoes not depend upon the planet's speed. Thus condition IV involves only $e_{1}$ and not $e_{2}$. It might be asked whether a condition could be established that involves $e_{2}$ and not $e_{1}$. The answer is yes. For example, the condition that the planet have the correct linear (not apparent angular) speed at aphelion is $r\left(1-e_{2}\right)=a\left(1-e+\frac{1}{2} e^{2}\right)$ to second order in the eccentricities, and where $a$ is the semimajor axis of the elliptical orbit and $r$ is the radius of the circular orbit. Similarly, the condition that the planet have the correct linear speed at perihelion is $r\left(1+e_{2}\right)$ $=a\left(1+e+\frac{1}{2} e^{2}\right)$. The linear speed does not, of course, depend upon the location of the observer; thus the conditions do not involve $e_{1}$.

${ }^{25}$ This familiar result for the true anomaly as a function of time is derived in most intermediate-level mechanics textbooks, as well as in most introductions to celestial mechanics. There are, of course, a number of different paths to the same result. See, for example, J. B. Marion, Classical Dynamics of Particles and Systems (Academic, New York, 1970), 2nd ed., pp. 258-259. Or, see W. M. Smart, Textbook on Spherical Astronomy (Cambridge U. P., Cambridge, 1977), 6th ed., pp. 119-120.

${ }^{26}$ The Copernican expression for $\theta(t)$ does, however, differ from the Ptolemaic in order $e^{3}$ because of the slight departure of the Copernican orbit from circularity.

\title{
Oscillations of a suspended chain
}

\author{
Rick Bylsma, a) Nguyen $\mathrm{Ai}^{\text {, }}{ }^{\text {) }}$ and D. A. Van Baak \\ Department of Physics, Calvin College, Grand Rapids, Michigan 49506
}

(Received 22 October 1987; accepted for publication 25 January 1988)

The venerable problem is considered of finding the natural frequencies of small oscillations about the equilibrium configuration of a suspended chain, under the assumptions of a continuous, perfectly flexible, inextensible, and lossless chain, supported at two fixed endpoints in a uniform gravitational field. A variety of analytical, variational, and numerical methods gives results that are valid over the full range of chain span-to-length ratios, including the limiting cases of very slack and very taut chains. Some surprising predictions of the theory are confirmed by experimental data.

\section{INTRODUCTION}

The equilibrium configuration of a suspended length of chain, the catenary curve, is familiar to many as a practical illustration of the hyperbolic cosine function and serves as a favorite example for teachers of variational methods in mechanics. Despite the wide currency of this knowledge, however, the problem of small oscillations about this equilibrium configuration has been only rarely addressed and never fully solved. In this article, we describe the allowed modes of oscillation of a suspended chain, and by using a variety of analytic, variational, and numerical methods, compute the eigenfrequencies of the modes for a full range of chain configurations.

The motivations for this work range from the theoretical (vibration theory in one-dimensional continuum mechan- ics) to the practical (wind-excited oscillations in electric power lines) and finally rest in the esthetic attraction of the physical problem and the mathematics describing it.

The modern history of this subject (following its nineteenth-century development by Routh ${ }^{1}$ ) includes a semiempirical treatment by Pugsley, ${ }^{2}$ an experimental approach by Satterly, ${ }^{3}$ and theoretical papers by Saxon and Cahn, ${ }^{4}$ Goodey, ${ }^{5}$ and Smith and Thompson. ${ }^{6}$ The existing theoretical treatments are restricted to rather flat, tightly stretched equilibrium conditions of the chain. In this article, we will overcome those limitations, finding results valid over the full range of chain span-to-length ratios. We derive, in Sec. II, the equations of motion for a length of idealized chain, and consider in Secs. III and IV the out-ofplane and in-plane oscillatory modes, respectively. In Sec. $\mathrm{V}$, we present some experimental data, a comparison with theory, and our conclusions. 